\title{
Buprenorphine management in the perioperative period: educational review and recommendations from a multisociety expert panel
}

\author{
Lynn Kohan (D) ,' Sudheer Potru (D) , ${ }^{2,3}$ Antje M Barreveld (D) , ${ }^{4}$ Michael Sprintz, ${ }^{5}$ \\ Olabisi Lane, ${ }^{6}$ Anuj Aryal, ${ }^{7}$ Trent Emerick, ${ }^{8}$ Anna Dopp, ${ }^{9}$ Sophia Chhay, ${ }^{9}$ \\ Eugene Viscusi (D) ${ }^{10}$
}

- Additional supplemental material is published online only. To view, please visit the journal online (http://dx.doi.org/ 10.1136/rapm-2021-103007)

For numbered affiliations see end of article.

Correspondence to Dr Lynn Kohan, Divsion of Pain Medicine/dept of anesthesia, University of Virginia, Charlottesville, VA 22903, USA; LRK9G@hscmail.mcc.virginia. edu

Received 1 July 2021 Accepted 20 July 2021 Published Online First 12 August 2021

\section{Linked}

- http://dx.doi.org/10.1136/ rapm-2021-103057 - http://dx.doi.org/10.1136/ rapm-2021-103092

Check for updates

(C) American Society of Regional Anesthesia \& Pain Medicine 2021. No commercial re-use. See rights and permissions. Published by BMJ.

To cite: Kohan L, Potru S,

Barreveld AM, et al.

Reg Anesth Pain Med

2021:46:840-859.

\section{ABSTRACT}

Background The past two decades have witnessed an epidemic of opioid use disorder (OUD) in the USA, resulting in catastrophic loss of life secondary to opioid overdoses. Medication treatment of opioid use disorder (MOUD) is effective, yet barriers to care continue to result in a large proportion of untreated individuals. Optimal analgesia can be obtained in patients with MOUD within the perioperative period. Anesthesiologists and pain physicians can recommend and consider initiating MOUD in patients with suspected OUD at the point of care; this can serve as a bridge to comprehensive treatment and ultimately save lives.

Methods The Board of Directors of the American Society of Regional Anesthesia and Pain Medicine, American Society of Anesthesiologists, American Academy of Pain Medicine, American Society of Addiction Medicine and American Society of Health System Pharmacists approved the creation of a Multisociety Working Group on Opioid Use Disorder, representing the fields of pain medicine, addiction, and pharmacy health sciences. An extensive literature search was performed by members of the working group. Multiple study types were included and reviewed for quality. A modified Delphi process was used to assess the literature and expert opinion for each topic, with 100\% consensus being achieved on the statements and each recommendation. The consensus statements were then graded by the committee members using the United States Preventive Services Task Force grading of evidence guidelines. In addition to the consensus recommendations, a narrative overview of buprenorphine, including pharmacology and legal statutes, was performed.

Results Two core topics were identified for the development of recommendations with $>75 \%$ consensus as the goal for consensus; however, the working group achieved $100 \%$ consensus on both topics. Specific topics included (1) providing recommendations to aid physicians in the management of patients receiving buprenorphine for MOUD in the perioperative setting and (2) providing recommendations to aid physicians in the initiation of buprenorphine in patients with suspected OUD in the perioperative setting. Conclusions To decrease the risk of OUD recurrence, buprenorphine should not be routinely discontinued in the perioperative setting. Buprenorphine can be initiated in untreated patients with OUD and acute pain in the perioperative setting to decrease the risk of opioid recurrence and death from overdose.

\section{INTRODUCTION}

Currently, opioid use disorder (OUD), involving both prescription opioid medications and illicit opioids, is a public health crisis in the USA, having reached epidemic proportions in the past several years. ${ }^{1}$ A recent national survey estimates that at least 2.5 million people in the USA have OUD. ${ }^{2}$ Previous models of OUD treatment, primarily focused on psychosocial counseling and behavioral treatments, have been strengthened by the addition of pharmacological therapies in association with these psychosocial treatments; this was formerly referred to as medication-assisted treatment (MAT) and is now known as medication treatment of OUD (MOUD). ${ }^{3}$ MOUD has been studied at length, and there is strong evidence demonstrating improved outcomes, increased retention in treatment, and decreased morbidity and mortality in the OUD population treated with this therapy. ${ }^{4}$

Given these benefits, expansion of access to MOUD critically decreases morbidity and mortality from OUD and associated medical problems ${ }^{5}$ with positive downstream effects on healthcare resources and society. Unfortunately, despite the opioid epidemic having been declared a national emergency in October 2017, a significant treatment gap remains between the number of patients diagnosed with OUD and those receiving MOUD. This reasons for this gap are complex and include multiple barriers, including stigma, an insufficient number of buprenorphine prescribers available to provide outpatient treatment, ${ }^{6}$ inadequate insurance coverage, and low payor compensation. The COVID-19 pandemic has posed unique and dangerous challenges for patients with OUD, including higher OUD recurrence rates, more overdose fatalities, and worsening barriers to care. ${ }^{7}$ The US Centers for Disease Control and Prevention (CDC) reported that over 81000 drug overdose deaths occurred in the 12 months preceding May 2020, representing the highest number of overdose deaths ever recorded in a 12 -month period. ${ }^{8}$

Now more than ever, physicians, including anesthesiologists and acute pain specialists, should consider MOUD for patients with OUD.

The current definition of addiction as stated by the American Society of Addiction Medicine (ASAM) is as follows: 
Box 1 American Psychiatric Association criteria for OUD

\section{Impaired control:}

1. Opioids are often taken in larger amounts or over a longer period than was intended.

2. There is a persistent desire or unsuccessful efforts to cut down or control opioid use.

3. A great deal of time is spent in activities necessary to obtain the opioid, use the opioid, or recover from its effects.

4. Craving, or a strong desire or urge to use opioids.

Social impairment:

5. Recurrent opioid use resulting in a failure to fulfill major role obligations at work, school, or home.

6. Continued opioid use despite having persistent or recurrent social or interpersonal problems caused or exacerbated by the effects of opioids.

7. Important social, occupational, or recreational activities are given up or reduced because of opioid use.

Risky use:

8. Recurrent opioid use in situations in which it is physically hazardous.

9. Continued opioid use despite knowledge of having a persistent or recurrent physical or psychological problem that is likely to have been caused or exacerbated by the substance.

Pharmacological criteria:

10. Exhibits symptoms of tolerance (reducing effect with increasing dose). *

11. Exhibits symptoms of withdrawal (physiological symptoms due to absence of a substance typically used repeatedly). *

*10 and 11 do not apply to individuals taking chronic opioids under medical supervision.

[Addiction is] a treatable, chronic medical disease involving complex interactions among brain circuits, genetics, the environment, and an individual's life experiences. People with addiction use substances or engage in behaviors that become compulsive and often continue despite harmful consequences. ${ }^{9}$

Although the definition of 'addiction' has evolved over time, the most current clinical nomenclature for a substance-related addictive disorder is 'substance use disorder' (SUD), where the term 'substance' is replaced by the actual substance of abuse (eg, alcohol use disorder, cocaine use disorder, etc). According to the most recent edition of the American Psychiatric Association's Diagnostic and Statistical Manual of Mental Disorders, ${ }^{10}$ each SUD is defined by 11 criteria divided into 4 categories. The criteria for OUD are shown in box 1 .

A patient must meet at least one of the pharmacological criteria in addition to at least one other criterion from another category to be diagnosed with an OUD. Meeting two to three criteria constitutes mild OUD, four to five is moderate, and six or more is defined as severe. The severity of the disorder may have implications for treatment. ${ }^{10}$

Prevention efforts and treatment approaches for SUDs are generally as successful as those for other chronic diseases, ${ }^{11}$ which is important to highlight when combating stigma. Clinicians are responsible for treating common chronic diseases; unfortunately, despite estimates that SUDs affect $>20$ million American adults at some point in their lives, ${ }^{2}$ many clinicians do not have significant training or experience in the treatment of SUDs. In this context, it is critical that more frontline physicians are trained in at least diagnosis (if not basic treatment) of SUDs. Doing so will provide vulnerable individuals with appropriate care and/or expeditious referral to a physician with appropriate credentials and knowledge of ancillary resources. In addition, the chronic and relapsing/remitting nature of OUD indicates that lifelong treatment is often needed to care for patients adequately.

From the standpoint of physicians who specialize in anesthesiology and pain management, some evidence demonstrates that prescription opioid exposure in both the perioperative and chronic pain settings has contributed to increased incidence of both persistent opioid use and possible OUD. ${ }^{12} 13$ However, recent evidence does not suggest a linkage in opioid-naïve patients undergoing total knee arthroplasty. ${ }^{14}$ In those who have been diagnosed with OUD, treatment of pain with most full opioid agonists, such as oxycodone or morphine, puts already vulnerable patients at risk for recurrence or worsening of their active OUD. ${ }^{12} 13$

US Food and Drug Administration (FDA)-approved medications for OUD include methadone, buprenorphine, and naltrexone, all of which have been found to be similar with respect to effectiveness, ${ }^{15} 16$ with the caveat that the efficacy of naltrexone is comparable (in the short term) only if patients withdraw and abstain from opioid use for 7-10 days prior to initiation of therapy. Of these, only methadone and buprenorphine are opioid agonists, meaning that there is potential for benefit from the standpoints of both craving, withdrawal suppression, and analgesia with both medications. ${ }^{15}$ A Cochrane meta-analysis found no significant differences in retention in treatment between buprenorphine and methadone at medium or high doses. ${ }^{17}$ However, a recent National Institute on Drug Abuse study found a higher rate of retention in treatment for methadone, while urine-confirmed abstinence was similar between the two groups. ${ }^{18}$

Methadone, when prescribed for OUD, is challenging to manage. Methadone for treating OUD must be dosed daily at a specialized facility, referred to as an opioid treatment program or 'methadone clinic', and which requires special licensure to operate. ${ }^{19}$ In addition, methadone may increase the risk of overdose if the dose is raised too quickly or combined with other illicit drugs before tolerance has fully set in. ${ }^{20}$ On the other hand, for the reasons indicated shortly hereafter, the partial agonist buprenorphine can be safely and effectively prescribed by many physicians in office-based settings. ${ }^{21}$ Currently, prescription of buprenorphine, unlike methadone, does not require dispensing in a clinic requiring a special regulatory license, alleviating a significant barrier for physicians. However, buprenorphine prescription does require applying for an $\mathrm{x}$-waiver (if treating $>30$ patients in the first year) or a notice of intent (NOI) to obtain an $\mathrm{x}$-waiver without training, which will be further explained below. ${ }^{3} 19$ Furthermore, buprenorphine can be prescribed discreetly in an office setting, diminishing the stigma and social barriers that may be associated with methadone clinics. ${ }^{19}$

In the perioperative setting, where acute pain is more likely, methadone's long half-life carries additional risk factors, including respiratory depression, ${ }^{22}$ which weighs against its use as a first-line opioid to be initiated in the setting of acute pain. In addition, while methadone is the primary pharmacological treatment for OUD in the USA, access to methadone is restricted by federal law (The Narcotic Addict Treatment Act of 1974) to highly regulated treatment programs. ${ }^{19}$ Buprenorphine may also decrease respiratory rate; however, the decrease is usually not clinically significant. ${ }^{23}$ The pharmacological profile of 
buprenorphine includes low intrinsic activity toward mu receptors; in addition, buprenorphine is a partial mu agonist at the mu receptor. Because methadone is a full mu agonist, it carries more potential for misuse and less protection from overdose compared with buprenorphine. ${ }^{16}$ Clinicians should also be aware that QT-interval prolongation and serious cardiac arrhythmias (eg, torsades de pointes) have been reported with methadone. ${ }^{24}$ The respiratory depressant effects of methadone persist longer than its analgesic effects. The terminal elimination half-life of methadone also has considerable interindividual variability, generally reported as 8-29 hours, but values have ranged from 9 to 87 hours in postoperative patients, from 8.5 to 75 hours in opiatedependent patients, and up to 120 hours in outpatients receiving therapy for chronic malignant pain. ${ }^{24}$ Because of its unique pharmacological properties, buprenorphine has potential advantages over methadone for OUD, including less sedation, fewer withdrawal symptoms, and lower risk of toxicity at higher doses. ${ }^{19}$

Ultimately, the choice to initiate buprenorphine, methadone, or naltrexone for OUD should be made collaboratively with the patient and care team and be consistent with the patient's goals. This paper does not seek to advocate for one form of MOUD over another. However, while methadone and naltrexone are viable options for MOUD, a full discussion of these medications is beyond the intended scope of this document.

The purpose of this multisociety collaborative document, based on literature review and expert opinion, is to serve as an educational resource for physicians focused on recognizing and managing OUD in the perioperative period. Specifically, the document will provide information on buprenorphine pharmacology, the perioperative management of patients on buprenorphine for OUD, and the advantages of initiation of buprenorphine postoperatively in patients with suspected OUD. This document is not intended to serve as a comprehensive guideline for treatment of OUD. We recognize the many challenges of co-managing pain and OUD in hospitalized patients. Since anesthesiologists and pain physicians knowledgeable about opioid pharmacology and management, we are uniquely poised to lead collaborative efforts to adequately treat OUD and acute pain. After hospital discharge, identification of and collaboration with outpatient providers may permit patients with OUD to receive immediate and sustained treatment in the community from appropriately trained clinicians, enabling services that promote best practice, encourage retention in treatment, and reduce OUD recurrence risk, overdose, and possibly death. Accordingly, trying to establish a 'hand-off' to an appropriately trained and experienced outpatient prescriber is preferred. While immediate lack of such services (eg, 'hand-off') should not uniformly prohibit the initiation of buprenorphine in patients with OUD experiencing acute pain, lack of prospective trials in this arena necessitates use of clinical judgment and comfort.

\section{METHODS}

A multisociety working group to develop guidance for anesthesiologists and pain physicians on managing patients with OUD was

Table 1 Levels of evidence for guidelines and recommendations Magnitude of net benefit

\begin{tabular}{lllll}
\hline $\begin{array}{l}\text { Certainty of net } \\
\text { benefit }\end{array}$ & Substantial & Moderate & Small & Zero \\
\hline High & A & B & C & D \\
Moderate & B & B & C & D \\
Low & Insufficient & & & \\
\hline
\end{tabular}

convened after approval from the American Society of Regional Anesthesia and Pain Medicine Board of Directors in early 2020. Societies with a vested interest in OUD and pain medicine were identified and formal request-for-participation letters were sent to each society, who all approved involvement. Each society selected one or two members to serve on the working group based on their expertize, clinical experience, and academic interests (see online supplemental appendix A for a list of participating societies and representatives). While the majority of the members have backgrounds in anesthesiology and/or pain medicine, 4 members (of the 10 -member working group) carry active board certification in addiction medicine, including one representative from the ASAM.

The multisociety working group, later known as The Substance Use Disorder Ad Hoc Working Group, was tasked with the common goals of (1) identifying the need for perioperative guidance on management of buprenorphine for MOUD for anesthesiologists and pain management physicians; (2) providing an overview of basic pharmacology of buprenorphine and review of legal prescribing requirements; and (3) developing recommendations on the management or initiation of buprenorphine in patients with OUD in the perioperative period. Pertinent questions and a proposed format were developed by the multisociety working group chair based on input from the group members and modified during video conference calls.

Once consensus was reached on a topic, the multisociety working group chair performed edits, and the section was sent to the entire group for further review and correction. At onset, the multisociety working group decided that $\geq 75 \%$ agreement was required for consensus, although 100\% consensus was achieved for all recommendations. After the multisociety working group completed the recommendations, the document was sent to the boards of directors of each society for approval. The document was subsequently edited based on feedback from individual societies.

Search engines used during the compilations of literature included PubMed, Google Scholar, MEDLINE, and Cochrane Database of Systematic Reviews, as well as the reference section of certain manuscripts. Search terms included buprenorphine, MOUD, MAT, OUD, perioperative, buprenorphine initiation, and $\mathrm{X}$-waiver. Guidelines from organizations and institutions, such as the Vermont guidelines and ASAM were reviewed. Articles were also evaluated and screened by the multisociety working group for relevance. There were no limitations in the types of articles that were used to develop the recommendations, but the quality of each piece of evidence was evaluated by one or more members of the group.

A modified Delphi process was used to tabulate comments, incorporate recommended changes, and compile the answers towards consensus over a series of nine conference calls. Recommendations were graded on a scale from A to D or as insufficient according to the United States Preventive Services Task Force grading of evidence guidelines with the level of certainty graded as high, medium, or low (tables $1-3) .{ }^{25}$ This grading system was chosen based on its use in numerous pain management guidelines ${ }^{26-28}$ and its flexibility, which allows for high-grade recommendations in the absence of level 1 studies and for multiple grades of recommendations. ${ }^{29}$

\section{Rationale for OUD treatment}

Increased education is warranted for anesthesiologists and pain physicians to recognize and treat OUD

Medical management of OUD saves lives. ${ }^{30}$ Despite access to effective screening and treatment tools, rates of opioid-related hospitalizations and deaths from opioid overdose continue 


\begin{tabular}{|c|c|c|}
\hline Grade & Definition & \\
\hline A & $\begin{array}{l}\text { Our committee recommends this treatment, test, or strategy to improve outcomes. } \\
\text { There is high certainty that the net benefit is substantial. }\end{array}$ & Offer or provide this service. \\
\hline B & $\begin{array}{l}\text { Our committee recommends this treatment, test or strategy to improve outcomes. } \\
\text { There is high certainty that the net benefit is moderate or there is moderate } \\
\text { certainty that the net benefit is moderate to substantial. }\end{array}$ & Offer or provide this service. \\
\hline $\mathrm{C}$ & $\begin{array}{l}\text { Our committee recommends selectively offering or providing this treatment, test, } \\
\text { or strategy to improve outcomes to individual patients based on professional } \\
\text { judgment and patient preferences. There is at least moderate certainty that the net } \\
\text { benefit is small. }\end{array}$ & Offer or provide this service for selected patients depending on individual circumstances. \\
\hline D & $\begin{array}{l}\text { Our committee recommends against the intervention. There is moderate or high } \\
\text { certainty that the service has no net benefit or that the harms outweigh the } \\
\text { benefits. }\end{array}$ & Discourage the use of this service. \\
\hline 1 & $\begin{array}{l}\text { Our committee concludes that the current evidence is insufficient to assess } \\
\text { the balance of benefits and harms of the intervention. Evidence is lacking, of } \\
\text { poor quality, or conflicting, and the balance of benefits and harms cannot be } \\
\text { determined. }\end{array}$ & $\begin{array}{l}\text { Read the clinical considerations section of the Recommendation Statement. If the treatment } \\
\text { or service is offered, patients should understand the uncertainty about the balance of benefits } \\
\text { and harms. }\end{array}$ \\
\hline
\end{tabular}

to rise. $^{31}$ The National Academies of Sciences, Engineering, and Medicine recently published Medications for Opioid Use Disorder Save Lives, a report on the importance of MOUD. The report emphasized barriers to greater use of MOUD including stigma, inadequate education, and restrictive regulations. ${ }^{30}$ Stigma against those with OUD is common within the healthcare setting $^{32}$ and fewer than $10 \%$ of physicians have completed the previously required training to prescribe buprenorphine. ${ }^{33}$

Lack of sufficient clinical training to provide care for patients with OUD is also a significant barrier. Medical schools and residencies often do not provide training in managing OUD. As of 2008, only 12 medical schools required a separate SUD course, while 45 offered it as an elective. ${ }^{34}$ Studies suggest that early training in evidence-based treatment is associated with higher confidence and willingness to provide OUD treatment. ${ }^{35}$ Thus, it has been reported that one of the most effective strategies for addressing the treatment gap may be to require healthcare professionals to be trained in the screening, diagnosis, and treatment of OUD and that such training should not be specialty specific. $^{32}$ The National Academy of Medicine (NAM) therefore recommends that accreditation agencies require clinicians receive training in OUD. ${ }^{32}$

An additional barrier is insufficient coordination of care among specialists. ${ }^{35}$ Interprofessional collaboration between practitioners of anesthesiology, chronic pain, surgery, primary care, and addiction medicine, along with frequent communication, are likely to improve both outcomes in care and patient satisfaction. ${ }^{36}$ The NAM report calls on all clinicians to receive education and training and to work together to combat the devastating consequences of OUD. ${ }^{32}$ Anesthesiologists can heed this call to action and play a leading role in treating patients with OUD.

Insurance and payor barriers are also significant; insurance plans may not cover or may require preauthorization for coverage of OUD medications. ${ }^{36}$ Advocacy work needs to continue to reduce these barriers. A full discussion of payor coverage limits is beyond the scope of this article.

Importantly, patients may report an interest in starting MOUD in the hospital setting. ${ }^{31}$ To be most effective, however, systems promoting ongoing, long-term care with MOUD after hospitalization are needed, and these may be most effectively provided by an outpatient physician experienced in OUD management. Several studies have shown that hospital-based OUD treatment with ongoing treatment after discharge is effective in terms of increasing entry into treatment, improving retention in treatment, increasing completion of hospitalization, and reducing opioid use and readmission. ${ }^{31} 37$

Hospitalization has been found to provide a teachable moment for initiating OUD care. ${ }^{38}$ Thus, anesthesiologists and pain physicians are in a unique position to help identify, treat,

Table 3 Levels of certainty regarding net benefit

\begin{tabular}{|c|c|}
\hline Level of certainty & Description \\
\hline High & $\begin{array}{l}\text { The available evidence usually includes consistent results from well-designed, well-conducted studies in representative primary care populations. The studies assess } \\
\text { the effects of the treatment, test or other intervention on treatment or other relevant outcomes. The conclusion is therefore, unlikely to be strongly affected by the } \\
\text { results of future studies. }\end{array}$ \\
\hline Moderate & $\begin{array}{l}\text { The available evidence is sufficient to determine the effects of the intervention on outcomes, but confidence in the estimate is constrained by such factors as: } \\
\text { The number, size, or quality of individual studies; } \\
\text { Inconsistency of findings across individual studies; } \\
\text { Limited generalizability of findings to routine primary care practice; } \\
\text { High likelihood of bias; } \\
\text { Lack of coherence in the chain of evidence. } \\
\text { conclusion. }\end{array}$ \\
\hline Low & $\begin{array}{l}\text { The available evidence is insufficient to assess effects on treatment and other outcomes of interest. } \\
\text { Evidence is insufficient because of: } \\
\text { The limited number or size of studies; } \\
\text { Important flaws in study design or methods; } \\
\text { Inconsistency of findings across individual studies; } \\
\text { Gaps in the chain of evidence; } \\
\text { High likelihood of bias; } \\
\text { Findings not generalizable to routine primary care practice; } \\
\text { Lack of information on important outcome measures. More information may allow estimation of effects on treatment outcomes. }\end{array}$ \\
\hline
\end{tabular}


and/or refer perioperative patients with OUD. While the number of patients with SUD presenting in the perioperative period is unknown, approximately $10 \%-30 \%$ of hospitalized patients have an untreated non-alcohol SUD. ${ }^{39}$ Untreated SUD often complicates the inpatient course secondary to poor adherence to medical treatment plans, withdrawal, and early cessation of appropriate treatment (as one-third of patients with SUD leave against medical advice). ${ }^{1940}$ Additionally, the economic and societal impact of untreated SUD is staggering. ${ }^{41}{ }^{42}$ Furthermore, death rates among patients with OUD are highest (31.7 per 1000) within the first month following a hospital discharge. ${ }^{43}$ Starting buprenorphine has been found to be one of the most effective ways to save lives; the number need to treat to prevent one death with buprenorphine is $<3 .{ }^{44}$ Larochelle et al also reported buprenorphine treatment was associated with a $37 \%$ reduction in all-cause mortality during the year following a non-fatal overdose. ${ }^{44}$ This mortality reduction is larger than the reduction in mortality associated with treatment with any blood-pressure medication, diabetic medication, or statin, and also larger than the reduction associated with aspirin after an ST-segment elevation myocardial infarction. ${ }^{45}$

Anesthesiologists and pain physicians can help lead efforts to screen, intervene, and initiate MOUD, and refer patients to ongoing community-linked treatment. Given the high mortality and the scarcity of outpatient resources for OUD treatment, it is important to recognize and treat OUD among perioperative patients. Although coordinating adequate outpatient follow-up, insurance coverage, and discharge planning is preferable, these should not be absolute requirements for initiation of this critical medication during a hospitalization, given some evidence that short-term exposure may reduce mortality, ${ }^{46}$ decrease presence of illicit substances, ${ }^{46}$ and be beneficial from a harm reduction standpoint, ${ }^{44}$ even when long-term follow-up is not available. Establishing inpatient collaboration and alliances with community programs and outpatient primary care practices can assist in streamlining care after hospital discharge. The recently reduced barriers to buprenorphine prescribing may assist in this process, as innovative methods to increase the ability to assist patients with OUD are urgently needed. Universal SUD screening can be integrated into the perioperative space, and physicians can be educated in the basics of addiction medicine, diagnosis of SUD, basic pharmacology, and multidisciplinary SUD treatment approaches. ${ }^{32}$ In addition, barriers to treatment access can be explored and addressed. Education, awareness, and empathy are key to decreasing the stigma of this treatable disease. In addition, physicians play an integral role in decreasing rates of OUD by collaborating with and educating the surgical team and other inpatient colleagues.

\section{Summary}

Levels of OUD are high in the USA; thus, anesthesiologists and pain physicians will encounter patients with treated and untreated OUD within the perioperative period. The NAM encourages all physicians to screen for and treat OUD. Anesthesiologists and pain physicians can play an integral role in leading efforts to screen and treat OUD within the perioperative period.

\section{Pharmacology}

There are three FDA-approved medications for the treatment of OUD (table 4). ${ }^{47-52}$ This manuscript will focus on the use of buprenorphine in the treatment of OUD.

Buprenorphine is a long-acting, mixed opioid agonist and antagonist that can lower the potential for misuse of opioids, diminish withdrawal symptoms and cravings, and offer protection in overdose situations. ${ }^{20}$ Its antagonistic properties provide safeguards against respiratory depression and diminish the euphoric effects of short-acting opioids. ${ }^{53}$ Buprenorphine is available as a single agent or in combination with naloxone. Naloxone is combined with buprenorphine to serve as a deterrent to injection use; naloxone taken orally or sublingually has low pharmacological activity. ${ }^{54}$ Buprenorphine is available in multiple dosage forms and requires special consideration when selecting a formulation or transitioning to a different dosage form. When indicated for the treatment of chronic pain, buprenorphine is available in a twice daily buccal film or weekly transdermal patch. When indicated for the treatment of OUD, buprenorphine is approved for use parenterally as an extended-release subcutaneous injection and sublingually as a tablet or film (as a single agent or in fixed combination with naloxone) (tables 5 and 6). While manufacturers and experts historically have preferred the use of buprenorphine alone for induction, comparative evidence is lacking and either formulation (buprenorphine with or without naloxone) may be used. ${ }^{55}$ Buccal as compared with sublingual administration results in greater bioavailability of naloxone, which may precipitate withdrawal symptoms on induction. Therefore, when the combination of buprenorphine/ naloxone is used, sublingual application of the buprenorphine/ naloxone film is preferred for minimizing exposure to naloxone and reducing the risk of withdrawal during induction therapy. ${ }^{55}$

Unfortunately, there is often confusion associated with the pharmacological properties of buprenorphine, most notably with regard to its analgesic potential. Specifically, buprenorphine is often mistakenly thought of as a weak analgesic. ${ }^{56}$ It is also often feared that buprenorphine will reduce the effectiveness of full mu opioids resulting in inadequate analgesia. Buprenorphine was, in fact, originally developed as an analgesic. ${ }^{57}$ Some clinical studies report that buprenorphine has similar or greater analgesic efficacy and antihyperalgesic effects as full mu-opioid receptor agonists. ${ }^{58-61}$ With regard to receptor binding, when buprenorphine is administered in maintenance doses typically prescribed for the treatment of OUD, mu-opioid receptors may still be available for binding of full mu agonists, although more pharmacological data and studies are needed.

\section{Summary}

Various buprenorphine formulations are FDA-approved for either the treatment of OUD or pain. Buprenorphine formulations that are FDA-approved for the management of OUD are available only in milligram dosing while formulations approved for analgesia are available only in microgram dosing. Buprenorphine is a potent analgesic despite being a partial mu-opioid receptor agonist. While buprenorphine has a strong affinity for the mu-opioid receptor, opioid receptors remain free for binding of full $\mathrm{mu}$ agonists even at standard doses of buprenorphine. Thus, although prospective data are lacking, effective analgesia may be obtained both with buprenorphine and with concomitant use of full $\mathrm{mu}$ agonists while a patient is also receiving buprenorphine. However, treating acute pain with opioids alone may be challenging for high levels of postsurgical pain; maximizing nonopioid and non-pharmacological agents is essential.

\section{DISCUSSION}

\section{Buprenorphine for the perioperative patient}

Patients with OUD frequently have high rates of hospitalization and readmission, long lengths of stay, and escalating healthcare costs. ${ }^{31} \mathrm{~A}$ subset of patients with OUD may present for surgery 


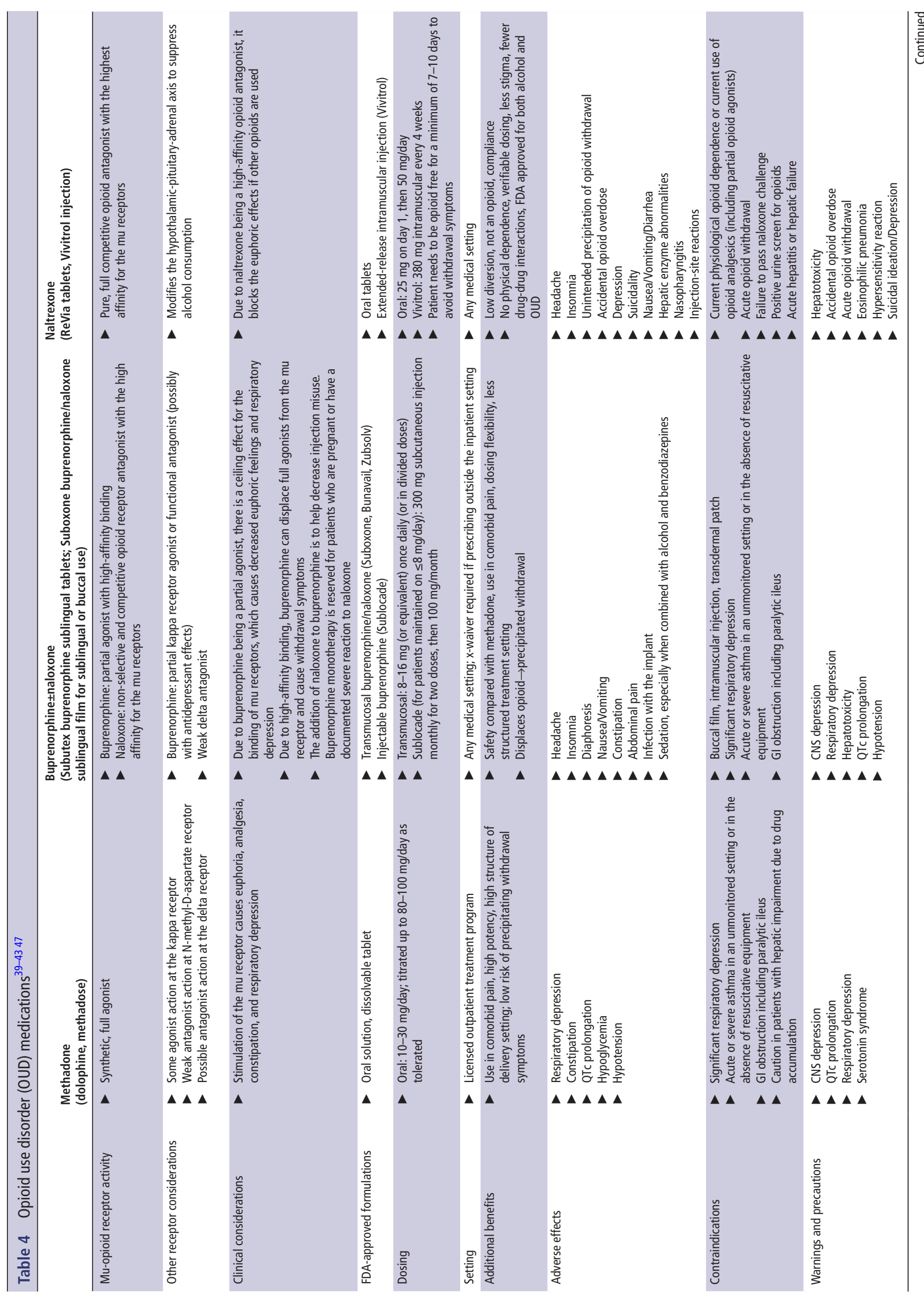




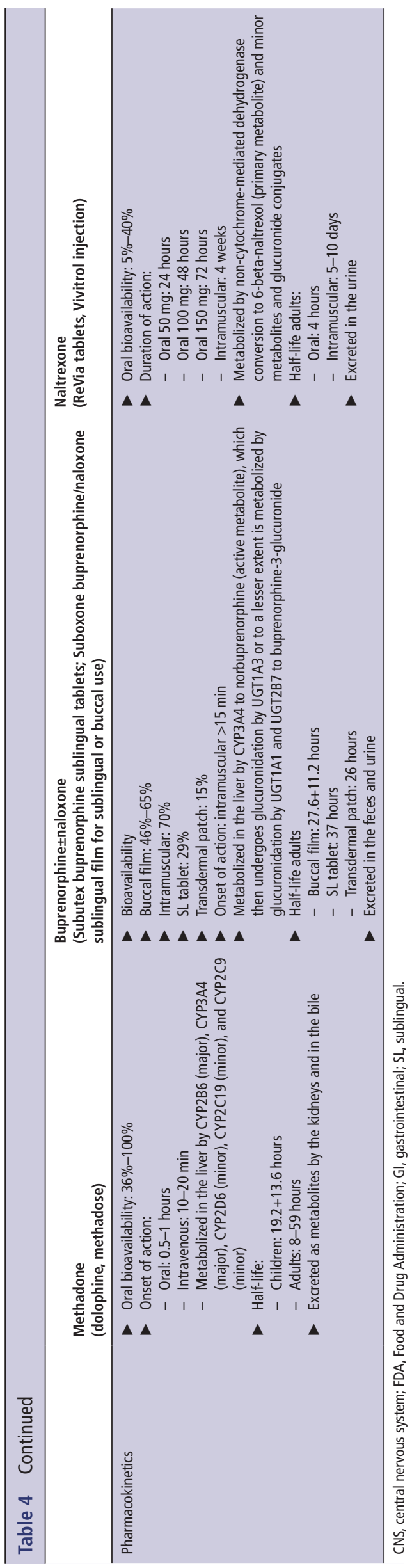

Table 5 FDA-approved buprenorphine formulations for MOUD and analgesia $^{50}$

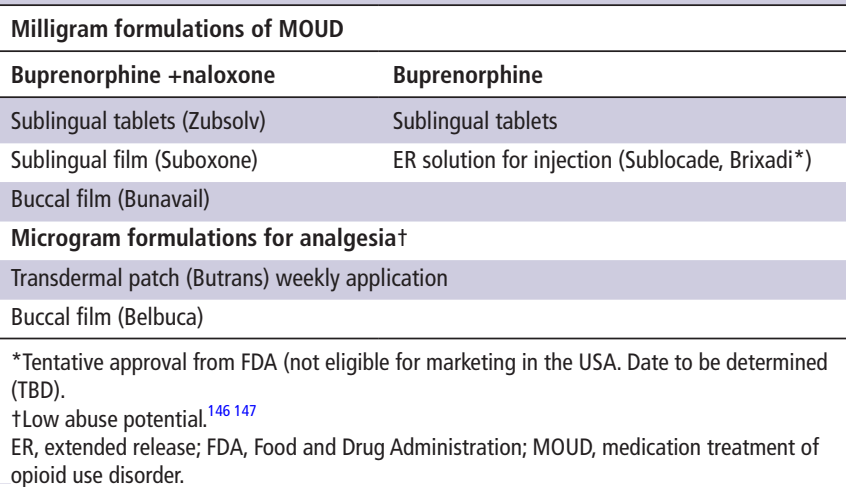

and have acute pain. Patients may have active untreated OUD or be in recovery and receiving MOUD. Each of these situations presents different challenges as well as opportunities for the anesthesiologist and acute pain service. ${ }^{62}$

\section{Perioperative management of a patient on buprenorphine for OUD}

In response to the opioid epidemic, increasing numbers of patients with OUD are being transitioned to buprenorphine from schedule II prescriptions or illicit opioids. ${ }^{63}$ From 2010 to 2016, annual prescriptions of buprenorphine formulations more than doubled. ${ }^{60}$ Thus, physicians are more frequently encountering patients on buprenorphine (with or without naloxone) who need surgery, necessitating more guidance as to how to care for patients with OUD. The perioperative period is a vulnerable time for patients with OUD. Patients with OUD may fear they will be treated unfairly or be judged by medical professionals during their hospitalization. Shame and stigma are also common. ${ }^{64}$ Additionally, patients worry about receiving inadequate pain relief, experiencing opioids withdrawal symptoms, and relapsing. ${ }^{65}$ Patients with OUD are at increased risk of recurrence following discharge from the hospital. ${ }^{43}$ Thus, a comprehensive preoperative evaluation, discussion of management of care, and communication with the primary inpatient service and/ or outpatient prescriber are essential.

Recommended preoperative evaluation guidelines for the management of perioperative pain include obtaining a history, performing a physical examination, identifying medications whose cessation may lead to withdrawal, and assessing for psychiatric and SUD comorbidities. ${ }^{66}$ Our working group recommends universal SUD screening if possible with validated screening tools for all preoperative patients. An example of easy-to-implement screening tools includes the single question screening test for drug use (National Institute of Drug Abuse (NIDA)-1 quick screen) ${ }^{67}$ which asks about substance use during the last 12 months. A positive screen can trigger a more detailed assessment tool such as the Drug Abuse Screening Test, ${ }^{68}{ }^{69}$ to

\begin{tabular}{lllll}
\hline Table 6 & Buprenorphine dosage formulations & \\
\hline & $\begin{array}{l}\text { Suboxone } \\
\text { SL }\end{array}$ & $\begin{array}{l}\text { Zubsolv } \\
\text { SL }\end{array}$ & $\begin{array}{l}\text { Sublocade } \\
\text { ER } \\
\text { subcutaneous }\end{array}$ & Bunavail buccal \\
\hline $\begin{array}{l}\text { Equivalent } \\
\text { dose of SL }\end{array}$ & $8 \mathrm{mg} / 2 \mathrm{mg}$ & $5.7 \mathrm{mg} / 1.4 \mathrm{mg}$ & $100 \mathrm{mg} / 0.5 \mathrm{~m}$ & $4.2 \mathrm{mg} / 0.7 \mathrm{mg}$ \\
\hline ER extended release: $\mathrm{SL}$ sublingual & & \\
\hline
\end{tabular}


assist in risk stratifying patients for additional support or referral for treatment when indicated.

Urine toxicology screens specifically testing for methadone, buprenorphine, and fentanyl may be helpful in addition to reviewing the prescription monitoring program if risk factors for OUD are present. ${ }^{66}$ It is important to note that anxiety about surgery and postoperative pain are significant stressors and may elicit a conditioned response, ultimately resulting in drug cravings. ${ }^{70}$ Exposure to prescription opioids and undertreated pain can also lead to cravings and OUD recurrence. ${ }^{71} 72$ Thus, it is imperative to discuss the risks and benefits of perioperative pain management modalities with a focus on OUD recurrence prevention. Careful discussion should ensue for patients receiving MOUD.

During the perioperative period, physicians may have concerns about the ability to treat postoperative pain given that a full mu-opioid agonist (hydromorphone, morphine, hydrocodone) is unable to displace buprenorphine. Buprenorphine has high affinity for the mu-opioid receptor, displaces full mu-opioid agonists, has an extremely long half-life (24-42 hours for sublingual or buccal administration; 26 hours for transdermal administration and 43-60 days for slow-release subcutaneous injection), is highly lipophilic, and slowly dissociates from the receptor. ${ }^{24}$ As such, oral buprenorphine takes 2-3 days to be eliminated from the body. While peak plasma concentrations increase with buprenorphine dose, the increase is not in direct proportion which results in a 'leveling off' of opioid effects, even with further dose increases. Buprenorphine is metabolized completely by the liver to norbuprenorphine, an active metabolite with some weak analgesic activity. Thus, previously published guidelines and expert opinion recommended discontinuing buprenorphine prior to anticipated pain or surgery. ${ }^{73} 74$ The 2004 Treatment Improvement Protocol released by the US Center for Substance Abuse Treatment stated that the administration of buprenorphine should generally be discontinued while patients are taking full mu-opioid agonist medications. ${ }^{74}$ This advisory influenced medical practice, leading to the commonplace discontinuation of buprenorphine prior to surgery. However, the recommendation was derived from case reports of difficult-to-treat acute pain in buprenorphine-maintained patients and may possibly reflect the challenge of managing already opioid-tolerant and opioid-dependent patients in need of analgesia as opposed to the consequences of buprenorphine itself. ${ }^{63}$ Furthermore, additional evidence suggests that buprenorphine in combination with full mu opioids can effectively treat perioperative or other acute pain. ${ }^{75-78}$

Even though buprenorphine has high affinity at the mu receptor, some receptors remain unoccupied and can continue to bind full $\mathrm{mu}$ agonists needed to treat acute pain in the perioperative period. ${ }^{79}$ Thus, perioperative management of buprenorphine is evolving from the traditional teaching of holding buprenorphine to 'open up receptors' to a consensus of continuing buprenorphine with or without naloxone through the perioperative period. Kornfeld et al evaluated the effectiveness of full agonist opioids in patients maintained on buprenorphine while undergoing major surgery. ${ }^{76}$ Physician assessment and patient self-report revealed adequate pain control with full opioid agonists and in some cases when multimodal treatment options were used. ${ }^{71}$ Mercadante et al were able to show the efficacy of opioid agonists in patients with cancer who were maintained on transdermal buprenorphine. ${ }^{80}$ It should be noted however that transdermal buprenorphine is a microgram formulation thus provides an overall lower dose of buprenorphine than are typically used for OUD. Furthermore, The ASAM's National
Practice Guideline for the Treatment of Opioid Use Disorder (2020 focused update) states, 'Discontinuation of methadone or buprenorphine before surgery is not required. Higher potency intravenous full agonist opioids can be used perioperatively for analgesia'. ${ }^{81}$ Additionally, it has even been reported that patients continued on maintenance doses postoperatively have a lower patient-controlled opioid analgesia requirement than those whose maintenance pharmacotherapy has been discontinued. ${ }^{82}$

Table 7 summarizes findings of perioperative management of patients on buprenorphine for MOUD. These studies comprised largely of case reports or series, cohort studies, or retrospective reviews. There were no randomized controlled trials.

While prospective trials are lacking, review of available literature suggests that buprenorphine (with or without naloxone) can be continued in the perioperative period while maintaining adequate analgesia. In a clinical practice advisory based on a review of evidence, Goel et al state, 'it is almost always appropriate to continue buprenorphine at the preoperative dose. Furthermore, it is rarely appropriate to reduce the buprenorphine dose'. ${ }^{83}$ As discussed below, individual considerations on perioperative buprenorphine maintenance dosing are a shared decision between the patient and the perioperative physicians and should be tailored to patient factors, anticipated pain severity, and the availability of regional anesthetic techniques, institutional resources, and professional expertize.

Continuation of buprenorphine in the perioperative period is further supported by evidence to suggest that it is harmful to discontinue buprenorphine. In fact, for some patients, discontinuation may even be fatal. ${ }^{84}$ Likelihood of harm is increased in patients prescribed buprenorphine for OUD as opposed to chronic pain. ${ }^{76}$

Bentzley et al determined that patients who discontinued buprenorphine maintenance treatment had a $>50 \%$ (range $50 \%-90 \%$ ) chance of OUD recurrence or, even worse, of death. ${ }^{82}$ Patients with OUD are also at an increased risk of inadvertent overdose when their maintenance treatment is discontinued due to a decrease in opioid tolerance and concurrent introduction of a full mu agonist. ${ }^{60}$ In addition, patients are often fearful of discontinuing buprenorphine. ${ }^{82}$

While there is growing support for the continuation of buprenorphine in the perioperative period, there is less agreement regarding the appropriate dose at which buprenorphine should be maintained. ${ }^{63}$ In addition to the studies in table 7 suggesting adequate pain postoperative pain control in patients maintained on buprenorphine, it may be beneficial to examine studies on receptor binding to help clarify the issue.

Human $\left[{ }^{11} \mathrm{C}\right]$-carfentanil positron emission tomography studies provide information into receptor binding occupancy at different buprenorphine doses. Study results exhibit some variability but consistently demonstrate some degree of opioid receptor availability, even at high buprenorphine doses. Greenwald et al demonstrated that when high doses of buprenorphine are used in heroin-dependent patients, there is a decrease in available mu-opioid receptors, an increase in buprenorphine levels, and a decrease in withdrawal symptoms, but also a decreased hydromorphone response overall. ${ }^{85}$ However, another study performed by the same group was able to show the percentage of available mu receptors at varying doses of buprenorphine: $71 \%-85 \%$ at $1 \mathrm{mg}, 53 \%-72 \%$ at $2 \mathrm{mg}, 36 \%-55 \%$ at $4 \mathrm{mg}$, $20 \%-35 \%$ at $8 \mathrm{mg}, 13 \%-24 \%$ at $12 \mathrm{mg}, 9 \%-20 \%$ at $16 \mathrm{mg}$, $4 \%-15 \%$ at $24 \mathrm{mg}$, and $2 \%-12 \%$ at $32 \mathrm{mg} .{ }^{85} 86$ The researchers concluded that patients can be maintained on buprenorphine with sufficient pain control from full mu-opioid agonists without worsened outcomes. Zubieta et al accessed mu-opioid receptor 
Table 7 Summary of case reports/series, reviews, and published guidelines on management of buprenorphine in the perioperative period

\begin{tabular}{|c|c|c|}
\hline Study & Study type & Findings and perioperative recommendations from literature \\
\hline Book SW et al $\left.\right|^{92}$ & Case report & $\begin{array}{l}\text { Patient successfully maintained on } 24 / 6 \mathrm{mg} \text { buprenorphine/naloxone up to the day of surgery. Additional sublingual doses of } \\
\text { buprenorphine aided in postoperative pain control. }\end{array}$ \\
\hline Marcucci C et al ${ }^{18}$ & Case report & $\begin{array}{l}\text { A man aged } 47 \text { years underwent total hip arthroplasty, patient on one tab sublingual buprenorphine every } 4 \text { hours (unknown } \\
\text { dose of tab). Buprenorphine discontinued day of surgery. Poor postoperative pain control. }\end{array}$ \\
\hline Brummett CM et al ${ }^{149}$ & Case report & $\begin{array}{l}\text { A man aged } 41 \text { years underwent postposterior lumbar spinal fusion. Patient on } 16 \mathrm{mg} \text { maintenance dose that was discontinued } \\
\text { day of surgery. Postoperative pain poorly controlled. } \\
\text { Recommendation: discontinue buprenorphine. }\end{array}$ \\
\hline Rodgman C et al ${ }^{150}$ & Case report & $\begin{array}{l}\text { A female patient aged } 29 \text { years underwent heart transplant. Patient on } 24 \mathrm{mg} \text { buprenorphine maintenance dose. Buprenorphine } \\
\text { discontinued } 12 \text { hours preoperatively. Postoperative pain control poor. } \\
\text { Recommendation: continue buprenorphine. }\end{array}$ \\
\hline
\end{tabular}

$\begin{array}{ll}\text { McCormick et al }{ }^{151} \quad \text { Case report } & \text { A man aged } 50 \text { years with McArdle's disease on } 24 \mathrm{mg} \text { buprenorphine/naloxone with exertional rhabdomyolysis requiring } \\ & \text { fasciotomies. Buprenorphine discontinued } 12 \text { hours prior to surgery. Postoperative pain poorly controlled. } \\ & \text { Patient with high intravenous hydromorphone requirements. } \\ & \text { Weaning and discontinuation recommendations provided. }\end{array}$

Chern SY et al ${ }^{93} \quad$ Case report Case report of a woman aged 37 years on buprenorphine with Chiari I malformation, hepatitis C, hypothyroidism. Patient had multiple urogynecological procedures, reported poor pain control with continuing buprenorphine perioperatively as well as poor pain control with stopping buprenorphine and bridging with oral hydromorphone for 5 days preoperatively. Recommendation: discontinue buprenorphine.

$\begin{array}{lll}\text { Israel JS et al }\left.\right|^{152} & \text { Case report } & \begin{array}{l}\text { A woman aged } 37 \text { years underwent bilateral mastectomy; home buprenorphine dose unknown. Buprenorphine discontinued } \\ \text { prior to surgery; postoperative pain poorly controlled. }\end{array} \\ \text { Huang A et al }{ }^{94} & \text { Case report } & \begin{array}{l}\text { A woman aged } 47 \text { years for Clagett window procedure for pulmonary aspergillosis. Suboxone } 16 \mathrm{mg} \text { two times per day } \\ \text { preoperative, and had poor postoperative pain control that led to postoperative taper of Suboxone with improvement in pain. } \\ \text { Managed on oral hydromorphone at time of discharge. } \\ \text { Recommendation: discontinue buprenorphine. }\end{array}\end{array}$

\begin{tabular}{|c|c|c|}
\hline Khelemsky Y et al ${ }^{153}$ & Case report & $\begin{array}{l}\text { A woman aged } 44 \text { years with cervical spine surgeries } 5 \text { days apart. Patient had reduced total intravenous anesthesia } \\
\text { requirements for the latter surgery when Suboxone ( } 8 / 2 \mathrm{mg} \text {, three times a day) was stopped after first surgery. } \\
\text { Weaning of Suboxone preoperatively is suggested. }\end{array}$ \\
\hline Silva MJ et a ${ }^{77}$ & Case report & $\begin{array}{l}\text { A man aged } 53 \text { years had one total knee arthroplasty on each knee approximately } 2 \text { years apart. Patient had better } \\
\text { postoperative pain control while on buprenorphine after first surgery (he was not on buprenorphine prior to second surgery). } \\
\text { Continuation of buprenorphine perioperatively is discussed. }\end{array}$ \\
\hline Jones et al $\left.\right|^{154}$ & $\begin{array}{l}\text { Case series } \\
\mathrm{n}=2\end{array}$ & $\begin{array}{l}\text { Buprenorphine* and methadone can be continued throughout the peripartum period without risk. Adequate pain control can be } \\
\text { achieved while patient is on maintenance dose with use of short-acting full mu agonists, acetaminophen and NSAIDs. }\end{array}$ \\
\hline
\end{tabular}

Kornfeld $\mathrm{H}$ et al ${ }^{76} \quad$ Case series $\quad$ Buprenorphine dose (ranging from 2 to $24 \mathrm{mg}$ preoperatively) maintained, decreased, or discontinued prior to surgery.
$\mathrm{n}=5,7$ cases (2 Pain well controlled in all patients regardless of discontinuing, maintaining, or increasing perioperatively/postoperatively. patients had 2 Recommendation: maintain stable buprenorphine dosing for patients who require major surgery. procedures)

\begin{tabular}{|c|c|c|}
\hline Mercadante $S$ et $a l^{80}$ & $\begin{array}{l}\text { Prospective } \\
\text { cohort study } \\
\mathrm{n}=29\end{array}$ & $\begin{array}{l}\text { Patients with cancer. Transdermal buprenorphine did not interfere with efficacy of intravenous morphine for breakthrough } \\
\text { analgesia in most patients. }\end{array}$ \\
\hline Hansen LE et al ${ }^{103}$ & $\begin{array}{l}\text { Prospective } \\
\text { cohort study }\end{array}$ & $\begin{array}{l}\text { Continue buprenorphine/naloxone or methadone for total knee and total hip arthroplasty. No significant difference between } \\
\text { groups. } \\
\text { Recommendation: buprenorphine can be continued. }\end{array}$ \\
\hline Höflich A et al ${ }^{155}$ & $\begin{array}{l}\text { Retrospective } \\
\text { cohort study }\end{array}$ & $\begin{array}{l}\text { Methadone and buprenorphine can be continued in the peripartum period with adequate postpartum pain control. } \\
\text { Limitation: methadone and buprenorphine patients combined into one group. }\end{array}$ \\
\hline Macintyre PE et al ${ }^{75}$ & $\begin{array}{l}\text { Retrospective } \\
\text { cohort study } \\
n=51\end{array}$ & $\begin{array}{l}\text { Pain relief and opioid requirements in first } 24 \text { hours compared between methadone and buprenorphine. } \\
\text { No difference between the two groups. No difference in pain control with continuing versus stopping buprenorphine. } \\
\text { Continuation of buprenorphine recommended: mean dose } 13.6 \mathrm{mg}( \pm 6.6 \mathrm{mg}) \text {. }\end{array}$ \\
\hline Vilkins AL et $a l^{78}$ & $\begin{array}{l}\text { Retrospective } \\
\text { cohort study } \\
n=273\end{array}$ & $\begin{array}{l}\text { Postcesarean section opioid requirements compared for patients on methadone or buprenorphine. No significant differences in } \\
\text { oral morphine equivalents in methadone or buprenorphine groups. } \\
\text { Conclusion: buprenorphine will not interfere more than methadone for postcesarean section pain management. }\end{array}$ \\
\hline Goel A et a $\left.\right|^{83}$ & Systematic review & $\begin{array}{l}\text { No evidence against continuing buprenorphine in the perioperative period, especially when dose is }<16 \mathrm{mg} \text {. There should be } \\
\text { strong rationale for stopping buprenorphine prior to surgery, especially in patients with recent history of OUD. }\end{array}$ \\
\hline Warner NS et $a^{34}$ & Systematic review & $\begin{array}{l}\text { Non-emergent: low pain expected: continue home buprenorphine dose; moderate-to-high pain expected: continue } \\
\text { buprenorphine if taking } \leq 8 \mathrm{mg} \text {, moderate-to-high dose-consult buprenorphine provider, may continue current home dose or } \\
\text { decease to } 8-12 \mathrm{mg} \text { prior to surgery } \\
\text { Emergent: low dose }(\leq 8 \mathrm{mg}) \text { continue current dose; high dose: continue current dose, use multimodal techniques, if pain } \\
\text { inadequately controlled consult buprenorphine provider and possibly decrease dose to } 8-12 \mathrm{mg} \text {. }\end{array}$ \\
\hline Mehta D et al/156 & Systematic review & $\begin{array}{l}18 \text { publications; no clear benefit to bridging or stopping buprenorphine; failure to restart might pose concerns for OUD } \\
\text { recurrence. } \\
\text { Recommendation: continue buprenorphine for OUD perioperatively; use interdisciplinary approach with multimodal analgesia. }\end{array}$ \\
\hline Alford DP et al ${ }^{157}$ & Review & $\begin{array}{l}\text { Multiple perioperative recommendations given including continuing buprenorphine and adding short-acting opioids, dividing the } \\
\text { daily dose of buprenorphine to every 6-8 hours. Discontinue and treat with short-acting opioids or convert to methadone. }\end{array}$ \\
\hline
\end{tabular}




\begin{tabular}{|c|c|c|}
\hline Study & Study type & Findings and perioperative recommendations from literature \\
\hline Childers JW et al ${ }^{158}$ & Review & $\begin{array}{l}\text { Mild/moderate pain: consider treating with buprenorphine alone or adding short acting full mu agonists as needed. } \\
\text { Hold buprenorphine and start short-acting full mu agonists if expecting moderate/severe pain. Replace buprenorphine with } \\
\text { methadone if prolonged pain is expected. Adjuvant analgesics and regional techniques should be employed. }\end{array}$ \\
\hline Bryson $\mathrm{EO}$ et al ${ }^{159}$ & Review & When possible, evaluate patient to see if buprenorphine can be discontinued 72 hours prior to surgery. \\
\hline Sen S et $a l^{160}$ & Review & $\begin{array}{l}\text { Discontinue buprenorphine } 72 \text { hours before surgery. Replace buprenorphine with methadone. } \\
\text { Anticipate additional opioid doses for pain control. }\end{array}$ \\
\hline Anderson TA et $a l^{73}$ & Review & $\begin{array}{l}\text { Patients can be stratified into urgent versus elective surgery; consider stopping buprenorphine for } 24-72 \text { hours if elective surgery } \\
\text { with moderate-to-severe postoperative pain. } \\
\text { Consider adjuvants: NSAIDs, membrane stabilizers, acetaminophen, local anesthetics, and regional anesthesia. }\end{array}$ \\
\hline Jonan $\mathrm{AB}$ et $\left.a\right|^{161}$ & Review & $\begin{array}{l}\text { Mild postoperative pain: continue buprenorphine without taper. } \\
\text { Moderate pain: discontinue buprenorphine } 3-5 \text { days before procedure, consider high-dose intravenous full-mu agonist patient } \\
\text { controlled analgesia. Severe pain: discontinue buprenorphine } 3-5 \text { days before procedure; consider full mu agonist to prevent } \\
\text { withdrawal. } \\
\text { Use non-opioids, regional anesthesia, and local anesthetics when possible. }\end{array}$ \\
\hline Ward EN et al ${ }^{65}$ & Review & Continue buprenorphine for mild-severe pain. Recommendation: multimodal analgesia. \\
\hline Harrison TK et $a l^{162}$ & Review & $\begin{array}{l}\text { Continue buprenorphine at home dose throughout perioperative period. If needed postoperatively, consider increasing } \\
\text { buprenorphine to control pain. }\end{array}$ \\
\hline Quaye AN et $a l^{88}$ & Review & $\begin{array}{l}\text { Mild pain: continue home dose of buprenorphine. Moderate-to-severe pain: reduce dose to } 16 \mathrm{mg} \text { up to the day before surgery } \\
\text { and } 8 \mathrm{mg} \text { on day of surgery and maintain } 8 \mathrm{mg} \text { daily. When surgical pain subsides, taper off full mu agonists and resume home } \\
\text { buprenorphine dose. }\end{array}$ \\
\hline Lembke A et a ${ }^{63}$ & Editorial & $\begin{array}{l}\text { Continue buprenorphine in the perioperative period for patients taking } \leq 12 \mathrm{mg} \text {; for those taking higher doses, taper to } 12 \mathrm{mg} \text {, } \\
2-3 \text { days prior to surgery. Anticipate higher-than-usual doses of short-acting full mu agonists for } 2-4 \text { days postoperative. }\end{array}$ \\
\hline $\begin{array}{l}\text { Berry P et al (Vermont } \\
\text { Guidelines) }\end{array}$ & Guidelines & $\begin{array}{l}\text { Decrease buprenorphine to } 8 \mathrm{mg} \text { sublingual on day of surgery; buprenorphine above } 10 \mathrm{mg} \text { will block opioid analgesics. Use } \\
\text { short-acting full mu agonists for postoperative pain; may need to use for longer period of time than anticipated. }\end{array}$ \\
\hline $\begin{array}{l}\text { ASAM National Practice } \\
\text { Guideline }^{81}\end{array}$ & Guidelines & $\begin{array}{l}\text { Discontinuation of methadone or buprenorphine is not required. Higher potency full mu agonists can be used perioperatively in } \\
\text { addition to the patients' regular dose. }\end{array}$ \\
\hline Goel et al ${ }^{91}$ & $\begin{array}{l}\text { Clinical practice } \\
\text { advisory/expert } \\
\text { opinion }\end{array}$ & $\begin{array}{l}\text { Continue buprenorphine at same dose perioperatively. If multimodal analgesia ineffective, consider decreasing buprenorphine } \\
\text { dose. }\end{array}$ \\
\hline $\operatorname{TIP} 40^{74}$ & $\begin{array}{l}\text { Treatment } \\
\text { improvement } \\
\text { protocol }\end{array}$ & Discontinue buprenorphine and use short-acting opioids (higher doses may be necessary). \\
\hline
\end{tabular}

*Patient was on $18 \mathrm{mg}$ buprenorphine.

NSAID, non-steroidal anti-inflammatory drug; OUD, opioid use disorder.

availability in three healthy controls, finding buprenorphineinduced dose-dependent opioid receptor availability reductions of $36 \%-50 \%$ at $2 \mathrm{mg}$ and $79 \%-95 \%$ at $16 \mathrm{mg}$ relative to placebo. ${ }^{87}$ In contrast, Quaye et al demonstrated that high doses (24-32 mg) of buprenorphine, as may be used for maintenance therapy, would result in little-to-no receptor availability, whereas moderate doses $(8-12 \mathrm{mg}$ ) would result in up to $20 \%$ receptor availability, which is still sufficient from an analgesic standpoint. ${ }^{88}$ Finally, Comer et al estimated that mu-opioid receptor availability was $21 \%-31 \%, 11 \%-22 \%$, and $6 \%-12 \%$ at 2,8 , and $32 \mathrm{mg}$ of buprenorphine, respectively. ${ }^{89}$ Collectively, these studies suggest that even at high doses (24-32 mg) of buprenorphine, some opioid receptors remain unoccupied. Furthermore, while we are unaware of any studies investigating the degree of analgesia obtained with full mu agonists with various levels of buprenorphine receptor occupancy, Comer et al suggest that buprenorphine $16 \mathrm{mg} /$ day did not fully block the reinforcing efficacy of $12.5 \mathrm{mg}$ and $25 \mathrm{mg}$ intravenous heroin. ${ }^{89}$ Similarly, Greenwald et al reported that buprenorphine $16 \mathrm{mg} / \mathrm{day}$ and 32 $\mathrm{mg}$ every other day did not fully block the reinforcing efficacy of $24 \mathrm{mg}$ intravenous hydromorphone. ${ }^{90}$

In conclusion, buprenorphine may produce similar clinical analgesic efficacy as a full mu agonist. ${ }^{58}$ There is some evidence that shows a relationship between mu receptor availability and withdrawal symptoms/heroin cravings. ${ }^{86}$ Unfortunately, the literature is lacking in terms of data that confirms degree of opioid receptor occupancy needed for analgesia for any mu agonist or partial agonist; thus, future studies are needed (eg, it is unknown what per cent of opioid receptors needs to be occupied by full or partial agonists in order to produce clinically noted analgesia and/or if the percent binding needed to produce analgesia differs among individuals).

In addition to understanding receptor occupancy, risk factors for exacerbation of OUD within the perioperative period should also be identified. In a 2019 systematic review, Goel et al suggest the following as potential risk factors: discontinuation of buprenorphine prior to surgery; introduction of a full mu agonist in place of buprenorphine prior to surgery; $<20$ months duration of buprenorphine for treatment of OUD; a positive urine drug screen within the last 20 months; discharge from the perioperative period without maintenance of buprenorphine and insufficient communication with the patient's outpatient buprenorphine prescriber. ${ }^{91}$

Thus, based on pharmacokinetic studies and other available literature, it is our recommendation that buprenorphine should not be routinely discontinued in the perioperative period. $^{76} 77$ 86-89 92-95 We do, however, recognize that there are no prospective clinical trials evaluating the optimal dose of perioperative buprenorphine. Prevailing recommendations based on preclinical data, site-specific experience, and consensus statements suggest adequate pain control in all but one patient taking $\geq 16$ mg sublingual buprenorphine. ${ }^{76} 779394$ Thus, our recommendation is to continue buprenorphine at the patient's home dose unless inadequate pain relief necessitates a change 
in the maintenance dose. With sufficient addiction and/or pain management or consultative expertize, a buprenorphine taper to $16 \mathrm{mg}$ could be considered for patients prescribed higher doses of buprenorphine (eg, $\geq 16 \mathrm{mg}$ ) and anticipated high postsurgical opioid requirements. We are aware, however, that for most healthcare systems, there may be insufficient access to OUD and pain medicine specialty consultations for guidance. ${ }^{6376}$ Thus, any needed change to a patient's outpatient buprenorphine regimen of buprenorphine, similar to other perioperative changes to outpatient medication changes, warrants a thorough discussion with both the patient and the primary buprenorphine prescriber regarding the associated risks and benefits, including the increased cravings or OUD recurrence during and after the hospitalization in conjunction with the need to achieve adequate analgesia.

It is important to understand that a multimodal analgesic regimen incorporating non-pharmacological therapies and nonopioid medications and interventions (eg, regional anesthesia) should be used in all patients continued on buprenorphine.

Pain in patients on maintenance buprenorphine (with our without naloxone) for OUD can be managed using multimodal therapies, similar to patients without OUD. As discussed earlier, a thorough conversation with the patient discussing multimodal treatment options is encouraged. Varying dosing strategies for acute or postoperative pain exist for patients prescribed buprenorphine for chronic pain or OUD. If possible, an acute pain consult should be obtained to help develop a safe perioperative management plan while minimizing OUD recurrence risk. ${ }^{65}$ Regional anesthesia as well as adjunct non-opioid medications and non-pharmacological alternatives should be used. ${ }^{95}$ Patient and family education and cognitive-behavioral approaches to manage anxiety, craving, and pain should be considered, particularly if opioid analgesics are prescribed. ${ }^{65}$ Discharge instructions should include safe use, storage, and disposal of opioids and communication with the surgical or hospitalist service.

As supported by most experts, we recommend the following strategies for treating acute pain in patients prescribed chronic buprenorphine. Figure 1 provides recommendations on a multimodal analgesic approach for patients taking buprenorphine (with or without naloxone) in the perioperative period. When using full $\mathrm{mu}$ agonists as part of the multimodal treatment plan, opioids with high affinity to the mu receptor such as fentanyl or hydromorphone should be used. Any patient treated with an increase or addition of opioids to aid in postoperative pain control needs to be provided a safe postoperative taper with specific instructions. Some evidence suggests that supplemental doses of buprenorphine be given in addition to continuing the patient's baseline buprenorphine dose to most effectively provide analgesia. ${ }^{76} 7792$ Collaboration between the patient's buprenorphine prescriber and/or addiction medicine team is always encouraged.

\section{Summary of recommendations \\ Preoperative planning}

Buprenorphine should not be routinely discontinued as adequate analgesia can be achieved (grade B, moderate level of certainty). Discontinuing buprenorphine can increase the risk of OUD recurrence or harm (grade B, moderate level of evidence). Current evidence suggests variation in recommendations with regard to tapering patients on high dose (>16 mg) of buprenorphine and in situations in which high levels of postoperative pain are anticipated; however, receptor availability studies and case reports suggest adequate analgesia can still be achieved even at high doses of buprenorphine. Thus, the working group recommends that, in addition to not routinely discontinuing buprenorphine prior to surgery, one should avoid tapering it perioperatively as well (grade B, moderate level of certainty).

\section{Postoperative pain}

Most available literature recommends the use of multimodal analgesia in the perioperative period in patients receiving buprenorphine for MOUD. Thus, the working group recommends that multimodal analgesia, including adjunctive medications and regional anesthesia techniques, should be used whenever possible (grade B, moderate level of certainty).

Additional evidence from opioid receptor binding studies and other literature review suggests that opioids can be administered in conjunction with buprenorphine to achieve adequate analgesia. Thus, it is the working group's recommendation to consider administration of full mu agonists (with high affinity for the mu receptor) (grade B, moderate level of certainty) or increased and/or divided doses of buprenorphine (grade C, low level of certainty) with close monitoring for uncontrolled postoperative pain if multimodal analgesia proves inadequate.

\section{Discharge planning}

Most studies on postoperative opioid use suggest providing a plan to taper the patient off postoperative opioids once the acute pain resolves. A taper plan can assist in minimizing withdrawal, decreasing postsurgical opioid use, and maximizing successful discontinuation of acute opioids once pain has resolved. ${ }^{96} 97$ Appropriate surgical prescribing and follow-up may ensure patients have tapered off postoperative opioids and acute pain management has been addressed. Similarly, if a full $\mathrm{mu}$ agonist is initiated or if buprenorphine is increased during the perioperative period, the working group recommends a postdischarge plan to taper off the full mu agonist or return to the preoperative maintenance dose of buprenorphine (grade A, moderate level of certainty). Additionally, length of recovery should be considered when prescribing full mu agonists on discharge. While providing full mu agonists with a taper plan may be reasonable for patients in stable recovery, caution is advised in those with active/recent illicit opioid use. Providing multiple daily prescriptions with 'do not fill' dates may be one technique to help mitigate the risk on this circumstance.

In addition, evidence from existing literature supports the working group's recommendation to engage in ongoing collaboration with the patient's outpatient buprenorphine prescriber (grade A, moderate level of certainty).

\section{Perioperative management of a patient with an untreated active} OUD

Substance use is prevalent among hospitalized patients: $36 \%$ use tobacco, 20\% use alcohol hazardously, and $8 \%$ use illicit drugs. ${ }^{98}$ While researchers have made strides in the development of brief interventions to decrease tobacco ${ }^{99}$ and alcohol abuse ${ }^{100} 101$ among hospitalized patients, advancements in the treatment of opioid misuse have been minimal. ${ }^{102}$ Patients with opioid dependence are at an increased risk of adverse health-related events, and thus, often seek care in emergency departments. ${ }^{103}$ Between 2004 and 2011, opioid-related emergency department visits increased $183 \%$. Nearly $25 \%$ of these visits resulted in hospital admission. ${ }^{104}$ Patients with OUD are approximately seven times more likely to be hospitalized than patients without OUD. ${ }^{105}$ These hospitalizations enable interventions to take place. While the number of patients with OUD presenting for surgery is 


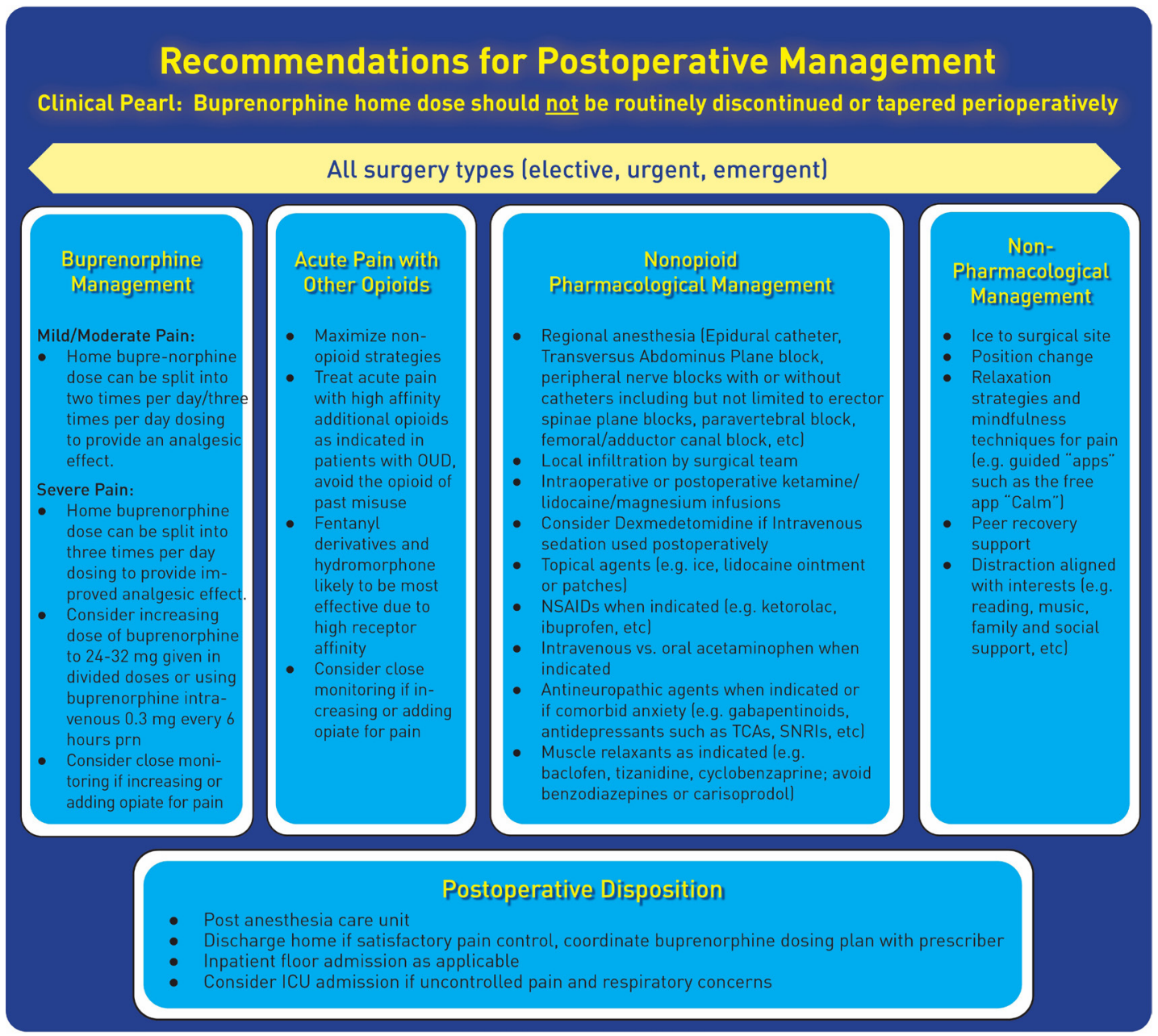

Figure 1 Recommendations for postoperative buprenorphine management. The recommendations above likely apply to the vast majority of healthcare systems, where addiction medicine/psychiatry services are not available to collaborate with anesthesia/acute pain services. In isolated clinical circumstances such as high-level academic medical centers with excellent integration of addiction and acute pain services, it is reasonable to consider a taper of buprenorphine in perioperative situations where extremely high levels of pain are anticipated and the admitting dose of buprenorphine is over $16 \mathrm{mg}$. If this is undertaken, it must be a shared decision between the patient, the buprenorphine prescriber, and the surgical team. An open discussion with the patient regarding possible drawbacks (including potential increase of craving and possibility of OUD recurrence) is necessary. This discussion should include a clear plan for discharge and follow-up with the primary prescriber. †The committee recognizes that not all clinicians will feel comfortable increasing the patient's home buprenorphine dose. Thus, increasing the home dose can be done after consulting with the patient's buprenorphine prescriber, the health system's addiction services team (if available), and the patient. ICU, intensive care unit; NSAID, nonsteroidal anti-inflammatory drug; OUD, opioid use disorder; SNRI, serotonin and norepinephrine reuptake inhibitor; TCA, tricyclic antidepressant.

unknown, this may provide an opportunity to initiate buprenorphine. ${ }^{106}$ Undertreated pain in conjunction with unaddressed OUD may result in less favorable outcomes, including premature discharge, worsening of underlying medical conditions, readmission, OUD recurrence, and overdose, both during the inpatient stay and immediately after discharge. Acute pain management necessitates special considerations and planning for patients with OUDs. ${ }^{65}$ Opioid withdrawal may also interfere with medical treatment; thus, withdrawal should be managed appropriately using a tapering schedule of opioid agonist substitution with methadone or buprenorphine. ${ }^{106} 107$ Furthermore, opioid withdrawal is considered a high-risk period that is associated with increased risk of opioid use, overdose, and death. ${ }^{60108-110}$

Studies suggest MOUD can be started safely during hospitalization, promoting engagement in outpatient SUD care and increased acceptance of MOUD. ${ }^{102} 104$ A study by Liebschutz et $a l$ and literature from the Substance Abuse and Mental Health Services Administration (SAMHSA) reported lower rates of illicit opioid use at a 6-month follow-up period among hospitalized patients who received buprenorphine induction and linkage to buprenorphine treatment on discharge. ${ }^{102} 111$ Liebschutz et al compared use of long-term MOUD between opioid-dependent hospitalized patients receiving buprenorphine induction and linkage to treatment versus detoxification. ${ }^{102}$ The buprenorphine-initiated group was found to have greater longterm use of MOUD compared with the detoxification group. In addition, D'Onofrio et al found emergency department-initiated buprenorphine with coordinated follow-up for continued treatment versus referral, with or without brief intervention, increased engagement in OUD treatment, reduced self-reported illicit opioid use, and decreased the use of inpatient OUD treatment services. ${ }^{101}$ Initiating buprenorphine must be a shared decision with the patient. Previous needs assessment studies have indicated that $67 \%$ of patients with active substance abuse would like to cut back or quit, and 44\% are interested in MOUD. ${ }^{31}$ Thus, given the desire of patients to seek treatment and the apparent 
efficacy of hospital-initiated treatment, it appears hospitalization may provide a moment for starting addiction treatment. ${ }^{38}$

It has also been reported that exposure to MOUD for even short periods of time increases survival. Studies report an instantaneous reduction in mortality after buprenorphine-assisted detoxification, even when access to long-term care and follow-up was not available. ${ }^{46}$ Every day, week, or month that a patient is receiving treatment is a period of time during which they have a reduced risk of overdose. ${ }^{112}$ Therefore, increasing pressure to make MOUD standard practice exists in some arenas. Leaders within emergency medicine (EM) have been called on by the Surgeon General and the CDC to aid in addressing the opioid epidemic by expanding patients' access to MOUD with buprenorphine. ${ }^{113}$ In addition, the state of Massachusetts recently mandated treatment of OUD in emergency departments. ${ }^{114}$ Furthermore, multiple studies report patients initiated on MOUD within the EM setting remained in long-term engagement of treatment for OUD. ${ }^{115}$

Unfortunately, many hospitals lack inpatient OUD services and pathways to care for and link patients to timely OUD care after discharge. ${ }^{116}$ Additional fears and/or limitations include the need for patients to abstain from opioid use for a period of time before starting buprenorphine/naloxone to provide adequate time for the elimination of systemic full mu opioids and avoiding precipitating opioid withdrawal with the use of a partial opioid agonist with high affinity for the opioid receptor. ${ }^{117}$

Thus, there is a need to create models that can improve care for patients with untreated OUD. One such approach is the induction of buprenorphine without requiring prior withdrawal symptoms, an approach used with increasing frequency in EM. ${ }^{118}$ Similar to the emerging evidence from EM, buprenorphine may be initiated safely in perioperative patients suspected of having an OUD.

Different approaches to inpatient induction are available and include waiting for opioid withdrawal (eg, Clinical Opioid Withdrawal Scale (COWS) 8 or 12) before giving buprenorphine; however, this is intolerable for some patients. New methods of initiating buprenorphine are evolving including microdosing and a method that describes a protocol essentially midway between the traditional method and the microdosing method. Microdosing, also known as the 'Bernese method', attempts to improve patient comfort by avoiding the need for withdrawal and minimizing the risk of precipitated withdrawal. ${ }^{119}$ Microdosing appears well tolerated by patients in case reports. ${ }^{119} 120$ The 'Bernese method' describes initiating patients on doses of $0.2 \mathrm{mg}$ of buprenorphine (compared with traditional induction doses of $\geq 2 \mathrm{mg}$ ) and slowly escalating while concurrently de-escalating the other opioid; however, a universally recommended protocol does not exist. ${ }^{119}$ While microdosing is becoming increasingly popular, a recent systematic review confirmed that publications are limited to case studies and no rigorous trials have been conducted. ${ }^{121} \mathrm{~A}$ recent study by Moe et al demonstrated that EM department-initiated buprenorphine/naloxone induction is feasible. ${ }^{121}$ While microdosing may therefore be an option in certain hospitalized patients, microdose initiations may take between 5 and 7 days, which may be longer than a patient's anticipated length of stay. Faster inductions with standard dosing have also been used successfully; however, there is currently no consensus for the optimal initial buprenorphine dose. ${ }^{122}$ For uncomplicated opioid withdrawal, most existing algorithms $^{122-126}$ suggest administering 4-8 mg buprenorphine sublingual and waiting approximately 1 hour. If withdrawal symptoms have improved, the physician may titrate an additional 4-8 mg as needed until cravings are suppressed. Discharge recommendations include documentation of opioid withdrawal and/or OUD as a diagnosis, providing a loading dose of $32 \mathrm{mg}$ if no $\mathrm{x}$-waivered provider is available to provide a prescription, and a $<7$-day prescription if an $\mathrm{x}$-waivered provider is available. ${ }^{126}$ If withdrawal symptoms do not improve after the initial dose, the following differential diagnoses should be considered: underlying illness mimicking withdrawal, such as influenza, diabetic ketoacidosis, and thyrotoxicosis; incomplete treatment withdrawal; side effects from buprenorphine itself; or too large a dose started too soon after opioid agonist (precipitated withdrawal). ${ }^{126}$ Uncomplicated withdrawal can be diagnosed using subjective report and objective signs.

\section{Withdrawal assessment}

\section{Subjective}

Patient reports feeling unwell: nausea, stomach cramps, body aches, yawning, goose bumps, vomiting, diarrhea, and/or tremor may be reported. ${ }^{99}$

\section{Objective}

At least one of the following symptoms is observed: restlessness, sweating, rhinorrhea, dilated pupils, watery eyes, tachycardia, yawning, goose bumps, diarrhea, or tremor.

Typical withdrawal onset occurs $>12$ hours after short-acting opioid; $>24$ hours after long-acting opioid; and $>48$ hours after methadone (can be up to $>72$ hours). ${ }^{101}$

A recent systematic review by Wolff et al assessed the current literature for EM-initiated buprenorphine. ${ }^{127}$ They identified 215 articles via various search engines. Of these, eight were selected based on relevance to the question 'In adult patients experiencing opioids withdrawal, is emergency roomadministered buprenorphine as effective for the management of opioid withdrawal compared with alternative management therapies'. They concluded that there were no level A recommendations. Buprenorphine or methadone are more effective options compared with non-opioid-based management strategies, such as adrenergic agonists and antiemetics; thus, using these medications for initiating treatment for opioid withdrawal in the emergency department is a level $\mathrm{B}$ recommendation. The authors recommended utilization of buprenorphine as opposed to methadone as a level $\mathrm{C}$ recommendation (box 2). ${ }^{128}$

In contrast to expert recommendations that advise waiting until mild-to-moderate withdrawal symptoms occur before initiating buprenorphine, ${ }^{129}$ a recent case series by Patel et al suggests that buprenorphine may be initiated prior to experiencing overt withdrawal. ${ }^{106}$ Patel et al provide a protocol for initiating buprenorphine for postoperative pain in patients with OUD in the perioperative period. ${ }^{106}$ Buprenorphine was initiated 4 hours after the last full mu opioid was given to allow for a washout period. The time frame was chosen based on the halflife (close to 4 hours) ${ }^{130}$ and general prescribing frequency (every 4-6 hours for pain) of oxycodone, the most commonly used full $\mathrm{mu}$ agonist at their institution. Intravenous hydromorphone, also commonly used for postoperative pain, has an even shorter half-life, ${ }^{131}$ with the expectation that initiating buprenorphine would not precipitate acute withdrawal. In the study by Patel et al, patients were provided $2 \mathrm{mg}$ sublingual buprenorphine as needed for pain every 2 hours during the first 24 hours for a maximum of 12 doses $(24 \mathrm{mg}) .{ }^{106}$ Additional full $\mathrm{mu}$ agonists were not provided. Three patients experienced mild withdrawal symptoms, such as nausea and diarrhea, and two patients had no subjective signs of withdrawal. Two patients were evaluated for the COWS; one had a score of 8 and the other 0 . Patients were 
Box 2 Levels of recommendation as defined by Hatten et $\left.\mathrm{a}\right|^{128}$

Level A recommendation

Generally accepted principles for patient care that reflect a high degree of scientific clinical certainty (eg, based on evidence from one or more class of evidence I or multiple class of evidence II studies demonstrating consistent effects or estimates.

\section{Level B recommendation}

Recommendations for patient care that may identify a particular strategy or range of strategies that reflect moderate scientific certainty (eg, based on evidence from one or more class of evidence II studies or multiple class of evidence III studies demonstrating consistent effects or estimates.

\section{Level C recommendation}

Recommendations for patient care that are based on evidence from class of evidence III studies, or in absence of any adequate published literature, based on consensus.

Reprinted with permission Hatten et al. ${ }^{128}$

${ }^{*}$ Note that the authors used different level of evidence and grading criteria than that used by the authors of this manuscript.

typically discharged on a dose of $8 \mathrm{mg}$ two times per day or 16 $\mathrm{mg}$ daily. Mean pain scores before and after buprenorphine initiation were $5.2 \pm 2.9$ vs $4.7 \pm 2.9(\mathrm{p}=0.4)$, respectively, suggesting that standard doses of full mu agonists were not essential to achieving adequate pain control. However, pain control cannot be attributed to buprenorphine alone as multimodal analgesia, including ketamine, was provided. On discharge, patients were linked to an outpatient buprenorphine provider. The study assessed the number of patients who filled their buprenorphine prescriptions after discharge (five out of seven); similar to strategies employed by internal medicine physicians and psychiatrists. ${ }^{132} 133$

Based on available evidence from hospital and EM inductions as well as the working group's expert opinion, prior to hospital discharge, buprenorphine may be initiated in small doses, similar to those used in the study by Patel et al ${ }^{106}$ which should maintain adequate analgesia without precipitating severe opioid withdrawal. Collaboration with the acute pain service or addiction specialist team is recommended if available, but absence of these services should not absolutely prohibit initiation. When considering initiating buprenorphine, it is important to first confirm that buprenorphine therapy is indicated. Many patients will divulge symptoms of OUD if asked. Screening tools such as the Opioid Risk Tool, Screener and Opioid Assessment for Patients with Pain, Current Opioid Misuse Measure, and Addiction Behaviors Checklist have been used successfully in $\mathrm{EM}^{134}$ and thus may be used within the perioperative setting.

It is the working group's recommendation to initiate buprenorphine at a lower dose than suggested in many EM protocols. ${ }^{122-126}$ Starting at a lower dose may lessen the risk of precipitated withdrawal and enable initiation within 4-6 hours of the last short-acting full mu-opioid agonist and within 1-2 hours of intravenous full mu agonist opioids. Adjuvant medications may be used, if necessary, to lessen withdrawal symptoms (table 8).

Figure 2 presents the working group's recommendations on starting buprenorphine in this manner. It is preferable that all patients initiated on buprenorphine be referred to an addiction
Table 8 Modalities used to treat opioid withdrawal symptoms

\begin{tabular}{|c|c|}
\hline $\begin{array}{l}\text { Withdrawal } \\
\text { symptoms }\end{array}$ & Management strategies \\
\hline Anxiety/Restlessness & $\begin{array}{l}\alpha_{2} \text {-Adrenergic agonists (eg, clonidine) } \\
\text { Relaxation, stress reduction strategies }\end{array}$ \\
\hline Insomnia & $\begin{array}{l}\text { Sleep aids (eg, trazodone, melatonin, hydroxyzine) } \\
\text { Relaxation strategies }\end{array}$ \\
\hline Musculoskeletal pain & $\begin{array}{l}\text { Acetaminophen } \\
\text { Ibuprofen }\end{array}$ \\
\hline $\begin{array}{l}\text { Gastrointestinal distress } \\
\text { (nausea, vomiting, } \\
\text { diarrhea) }\end{array}$ & $\begin{array}{l}\text { Oral hydration and electrolyte replenishment } \\
\text { Hycosamine } \\
\text { Antiemetics (eg, ondansetron) } \\
\text { Antidiarrheals (eg, loperamide) }\end{array}$ \\
\hline
\end{tabular}

medicine specialist, primary care provider, or other community resource. Until a relationship with a local provider is established, it has been recommended to call any available community buprenorphine prescriber before starting buprenorphine ${ }^{122}$ in the hospital if able; however, our group does not recommend delaying necessary treatment if a community provider is unavailable. A buprenorphine prescriber locator is available on the SAMHSA website to facilitate establishing community linkage if needed. ${ }^{135}$ A needs assessment study within the EM literature highlighted the discordant priorities of the emergency room (rapid and flexible referral process) and the community prescribers offering buprenorphine; this emphasizes the need for increased availability and accessibility to MOUD on demand and the importance of communication between EDs and community prescribers of MOUD. ${ }^{136}$ To help accomplish this goal, they recommend that a date, time, and follow-up location should be provided whenever possible in order to ensure a smooth transition. ${ }^{136}$ Patients can alternatively register with an anonymous buprenorphine provider matching system that facilitates patientprovider contact. ${ }^{137}$ Perioperative physicians can also use lessons learned from the EM literature to help facilitate processes that enable hand-offs of care, including use of social workers to help coordinate outpatient MOUD referrals without increasing physician workload or costs. ${ }^{138}$ In a retrospective, cohort, singlecenter study, Kelly et al describe a social work-driven emergency department initiation of buprenorphine program with referral to community MOUD providers. Patients with OUD presenting to the ER were identified by patient self-report, standardized nursing screening, or emergency department provider concern. All patients who were identified in this manner received an urgent social work consult to explore willingness to seek treatment for OUD. The social workers developed individual plans to help link patients to appropriate community prescribers. ${ }^{138}$

A recent review by Martin et al further describes models to establish maintenance treatment on discharge; including the Substance Abuse Services and Referral to Treatment model, bridge model, and emergency department-bridge model. ${ }^{139}$ These models may provide useful guidance in implementation and logistical details to support health systems in better addressing OUD in their communities.

We recognize that barriers may exist due to the patients' insurance or lack of insurance and difficulty finding a suitable outpatient buprenorphine prescriber, which may prevent handing-off the patient's care. While a hand-off is preferable and strongly recommended, short-term treatment without follow-up has been demonstrated to reduce mortality, ${ }^{46}$ decrease presence of illicit substances,${ }^{140}$ and increase harm reduction ${ }^{44}$; thus, lack of such resources should not uniformly prohibit initiation of buprenorphine in this setting. Any physician who decides to prescribe 


\section{How to Initiate Inpatient Buprenorphine for a Patient with} Suspected Opioid Use Disorder in the Perioperative Period

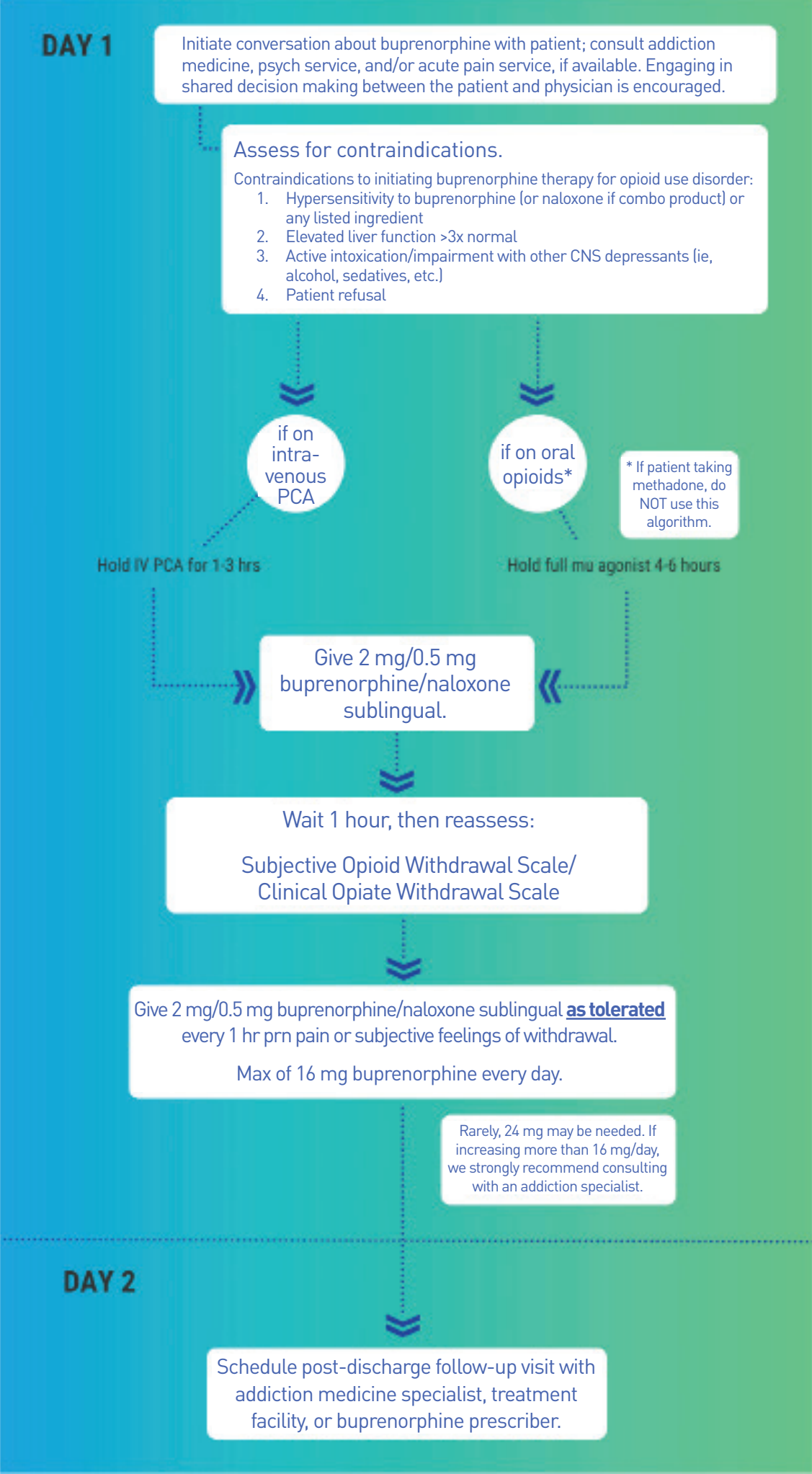

Figure 2 How to initiate buprenorphine for a patient with suspected opioid use disorder (OUD) in the perioperative period. *We do not recommend using this algorithm (eg, initiating buprenorphine) in patients with chronic pain who are currently being prescribed long-acting opioids in the perioperative period. Clinical Opioid Withdrawal Scale (COWS): see online supplemental appendix B. Subjective Opioid Withdrawal Scale (SOWS): see online supplemental appendix C. CNS, central nervous system; PCA, patient-controlled opioid analgesia. 
Box 3 Strategies for in-hospital management of patients with opioid use disorder (OUD)

\section{Screening}

- Most patients will disclose their substance use history when asked.

- Urine toxicology is generally not required but may be useful, particularly in identifying patients who have recently taken methadone, which may make initiation of buprenorphine more difficult.

- Some clinics may require a positive urine toxicology for opioids prior to medication treatment of opioid use disorder (MOUD) treatment, which may be important when linking to community care.

\section{Medication administration}

- See committee recommended algorithm. Figure 2 (inpatient buprenorphine initiation).

- The Clinical Opioid Withdrawal Scale may be a useful tool to help physicians and nurses who are new to diagnosing opioid withdrawal. Use of subjective signs and objective signs of withdrawal is also valid.

- May include naloxone for respiratory rate $<8$ breaths per minute.

\section{Discharge planning}

- If able, provide buprenorphine prescription for OUD as bridge to community-linked treatment. Requires $x$-waivered physician.

- If an $\mathrm{x}$-waivered provider is not available, ensure immediate linkage to community clinic providing MOUD care.

- Provide naloxone prescription or information at discharge.

- Provide information for follow-up to community-linked clinic.

Adapted from information at https://cabridge.org/ ${ }^{142}$

buprenorphine when a 'hand-off' is not possible should consider the risks and benefits unique to the clinical situation, including their own comfort level and education, as well as balancing the risks of prescribing a full mu agonist on discharge versus buprenorphine in regard to OUD recurrence prevention and analgesic control.

Tools to aid in the development of protocols for evidencebased treatment of OUD exist, including guidance on screening, medication administration, and preparation for discharge. ${ }^{141}$ Order sets can be developed to aid physicians in the process. The Ca Bridge to Treatment program issued a Best Practices: Inpatient and Order Set Guideline, which is a useful resource (box 3). ${ }^{142}$

Pathways that guide management of patients from hospital to community OUD treatment are likely to be absent in many hospital systems. A recent needs assessment study employed the use of 'in reach' liaisons-community SUD treatment staff who perform in-hospital assessments to triage and coordinate care across systems. ${ }^{31}$ We recognize that these types of 'in reach' liaisons may not be feasible; thus, we recommend coordinating with social workers to help facilitate transition to community basedcare where possible. It is also important to note that the link to care should include a variety of options, including MOUD, and abstinence programs, office-based treatment, intensive outpatient treatment, and residential levels of care, thereby giving patients many options. ${ }^{31}$

\section{Recommendation}

Patients with suspected OUD can be approached and educated about the benefits of initiating buprenorphine postoperatively. Current literature, largely from the field of EM, has demonstrated that initiation of buprenorphine for patients with OUD results in decreased use of illicit substances and greater retention in OUD treatment programs. Safe initiation of buprenorphine with linkage to a community provider was described in several available EM studies, as well as by an anesthesia-led team in a case series. Thus, based on both review of this literature and expert opinion, it is the working group's recommendation that, when possible and clinically indicated, anesthesiologists/pain physicians can consider recommending or starting buprenorphine for postoperative analgesia in patients with suspected OUD, using available social work or ancillary services to help facilitate linkage to outpatient buprenorphine prescribers when possible (grade of evidence B, moderate level of certainty). However, per updated Department of Health and Human Services (HHS) guidelines, buprenorphine treatment can now be legally given without concomitant ancillary treatment. ${ }^{143}$ Thus, one additional recommendation, based on physician expertize/ comfort, is that buprenorphine treatment can still be considered in circumstances in which follow-up/insurance coverage has not been fully established (grade of evidence C, low level of certainty). Buprenorphine, with its long half-life and partial agonism, is likely a safer alternative for discharge medication than full mu-opioid receptor agonists in patients with OUD, even without an established connection to an outside buprenorphine prescriber. Thus, in circumstances in which a warm hand-off has not been definitely established, the amount of buprenorphine prescribed can be consistent with appropriate postoperative discharge standards; however, a longer course of treatment could be provided, depending on the prescribing physician's comfort level. Initiating buprenorphine should always entail a shared decision-making process between the patient and the physician. The patient should also be given whatever resources are available within the local community to encourage continuation of SUD treatment, whether medical or psychosocial.

\section{Legal issues and $\mathrm{x}$-waivers}

Rules and regulations regarding the prescription of buprenorphine are confusing and may lead to decreased access to care. However, given recent data showing an increase in opioid-related overdose deaths in the year preceding August 2020, ${ }^{144}$ there has been a renewed emphasis at the federal level to increase access to MOUD. ${ }^{144}$ There have also been several recent legal changes and updates related to the $\mathrm{x}$-waiver certification process. On April 28, 2021, the Department of HHS announced that qualified providers who are state-licensed and registered by the DEA are exempt from the current $\mathrm{x}$-waiver certification training requirements. ${ }^{141}$ To qualify for the exemption, physicians must adhere to a limit of treating to no more than 30 patients at any one time, and they still must submit a NOI to obtain an $\mathrm{x}$-waiver to SAMHSA. These exemptions only apply to the prescribing of schedule III, IV, and V medications and not schedule II medications such as methadone for OUD. Furthermore, physicians can only treat patients under this exemption in states where they hold an active medical license.

Clinicians can order milligram (ie, MOUD) FDA-approved formulations of buprenorphine to hospitalized (perioperative) patients without applying for an NOI and can increase the dose if needed in order to 'maintain or detoxify a person as an incidental adjunct to medical or surgical treatment of conditions other than 
Box 4 Steps for obtaining $x$-waiver certification (if desiring to treat $>30$ patients)

1. Complete training. -8 hours hours for physicians.

- 24 hours for mid-level providers.

2. Apply to Substance Abuse and Mental Health Services Administration for ' $x$-waiver'.

- Training available at https://pcssnow.org/ medications-for-opioid-use-disorder/

addiction, or to administer or dispense narcotic drugs to persons with intractable pain in which no relief or cure is possible or none has been found after reasonable effort ${ }^{\text {, }}{ }^{144}$ Accordingly, patients admitted for conditions such as endocarditis related to drug use, osteomyelitis, abscess, and trauma related to injection drug use are all eligible for treatment with buprenorphine or methadone in the hospital setting. FDA formulations approved for the treatment of chronic pain, such as buprenorphine transdermal patch (Butrans) and the buccal formulation (Belbuca), cannot be prescribed for the treatment of OUD; however, they can be used for the treatment of analgesia in any setting without obtaining a $\mathrm{x}$-waiver. ${ }^{145}$

\section{X-waiver certification process}

$\mathrm{X}$-waiver certification is still required for physicians who wish to treat $>30$ patients at a time. The training for physicians involves 8 hours of continuing medical education and can be completed fully online free of charge (box 4).

\section{Summary}

$\mathrm{X}$-waivers are not required to prescribe/dispense buprenorphine while the patient is in the hospital or emergency department; however, they are required to provide a prescription for buprenorphine that a patient will fill at a pharmacy. The training to obtain an $\mathrm{x}$-waiver is waived when treating $<30$ patients and submitting an NOI to SAMHSA.

It is the working group's consensus to advocate for the elimination of barriers to prescribing buprenorphine for patients with OUD. We also advocate that in the interim physicians obtain education in MOUD and submit an NOI to obtain an $\mathrm{x}$-waiver.

\section{Consensus}

The presubmission version of these recommendations was sent to participating societies in March 2021, and approved by the American Society of Regional Anesthesia and Pain Medicine, American Academy of Pain Medicine, and the American Society of Health-System Pharmacists. The American Society of Anesthesiologists and the ASAM requested some additional edits which were included by the working group. There was $100 \%$ consensus among the committee members (coauthors) for each recommendation. All five societies supported the recommendations in the manuscript. These guidelines were approved en bloc by the American Society of Administrative Council and Committees on Pain Medicine (Acute and Chronic), but were not voted on by their Board of Directors.

\section{CONCLUSION}

OUD is a chronic debilitating disease that results in significant morbidity and mortality. Increasing the use of buprenorphine during the perioperative period is one important way to decrease morbidity and mortality, and anesthesiologists and pain physicians are uniquely qualified to lead this effort. Buprenorphine is an effective FDA-approved medication for the treatment of pain and OUD. While this document addresses numerous issues regarding the use and prescription of buprenorphine in the perioperative period, the overarching goals of this multisociety working group are to educate anesthesiologists and pain physicians and to encourage the use of evidence-based treatment options for OUD. Understanding the significant morbidity and mortality caused by OUD is critical for both individual patient care and our current public health crisis. This is a prime instance where our specialty can and should make a difference. This multisociety working group encourages physicians to learn more about this safe, efficacious, and underused treatment that can save lives. See online supplemental appendix 4 for summary of committee recommendations.

\section{Author affiliations}

'Division of Pain Medicine/Department of Anesthesia, University of Virginia, Charlottesville, Virginia, USA

${ }^{2}$ Atlanta VA Medical Center, Emory University School of Medicine, Atlanta, Georgia, USA

${ }^{3}$ Department of Anesthesiology, Emory University School of Medicine, Atlanta, Georgia, USA

${ }^{4}$ Department of Anesthesiology, Tufts University School of Medicine, NewtonWellesley Hospital, Boston and Newton, Massachusetts, USA

${ }^{5}$ Division of Geriatrics and Palliative Medicine, The University of Texas Health Science Center at Houston, Houston, Texas, USA

${ }^{6}$ Division of Pain Medicine, Department of Anestheisology, Emory University School of Medicine, Atlanta, Georgia, USA

${ }^{7}$ Cedar Recovery and Deparment of Anesthesiology and Pain Medicine, VA Tennessee Valley Healthcare System Nashville Campus, Nashville, Tennessee, USA

${ }^{8}$ Department of Anesthesiology and Perioperative Medicine, University of Pittsburgh

Medical Center, Pittsburgh, Pennsylvania, USA

${ }^{9}$ American Society Health System Pharmacists, Bethesda, Maryland, USA

${ }^{10}$ Thomas Jefferson University Sidney Kimmel Medical College, Philadelphia,

Pennsylvania, USA

Correction notice This article has been corrected since it published Online First. The author affiliations have been updated.

Twitter Lynn Kohan @kohanlynn and Sudheer Potru @SPotruD0

Acknowledgements The authors would like to thank Angie Stengel for her administrative assistance in coordinating conference calls and outreach to participating organizations. We would also like to thank Anne Snively and Athena Ermidis for their help with figure creation.

Contributors All authors contributed to the development, writing and revision of the manuscript.

Funding The authors have not declared a specific grant for this research from any funding agency in the public, commercial or not-for-profit sectors.

Disclaimer This document is based on literature review and expert opinion. It is not intended to establish standard of care.

Competing interests None declared.

Patient consent for publication Not required.

Provenance and peer review Not commissioned; externally peer reviewed.

Data availability statement All data relevant to the study are included in the article.

\section{ORCID iDs}

Lynn Kohan http://orcid.org/0000-0003-0407-806X

Sudheer Potru http://orcid.org/0000-0002-7081-1154

Antje M Barreveld http://orcid.org/0000-0003-1126-2797

Eugene Viscusi http://orcid.org/0000-0003-0260-4396

\section{REFERENCES}

1 Rudd RA, Seth P, David F, et al. Increases in drug and opioid-involved overdose deaths - United States, 2010-2015. MMWR Morb Mortal Wkly Rep 2016:65:1445-52.

2 Substance Abuse and Mental Health Services Administration. Key substance use and mental health indicators in the United States: results from the 2017 national survey 
on drug use and health. Available: https://www.samhsa.gov/data/report/2017nsduh-annual-national-report [Accessed 21 Mar 2021].

3 Bart G. Maintenance medication for opiate addiction: the foundation of recovery. J Addict Dis 2012:31:207-25

4 Sordo L, Barrio G, Bravo MJ, et al. Mortality risk during and after opioid substitution treatment: systematic review and meta-analysis of cohort studies. BMJ 2017;357:j1550.

5 Leslie DL, Ba DM, Agbese $E_{\text {, et }}$ al. The economic burden of the opioid epidemic on states: the case of Medicaid. Am J Manag Care 2019;25:S243-9.

6 Saitz R, Daaleman TP. Now is the time to address substance use disorders in primary care. Ann Fam Med 2017:15:306-8.

7 Volkow ND. Collision of the COVID-19 and addiction epidemics. Ann Intern Med 2020;173:61-2.

8 US Centers for Disease Control and Prevention. Overdose deaths accelerating during COVID-19, 2020. Available: https://www.cdc.gov/media/releases/2020/p1218overdose-deaths-covid-19.html [Accessed 10 Mar 2021]

9 American Society of Addiction Medicine. Definition of addiction. Available: https:// www.asam.org/quality-practice/definition-of-addiction [Accessed 21 Sep 2020].

10 American Psychiatric Association. Substance-Related and Addictive Disorders. In: Diagnostic and statistical manual of mental disorders. 5 edn, 2013.

11 American Society of Addiction Medicine. Patient resources. Available: https://www. asam.org/Quality-Science/resource-links/patient-resources [Accessed 5 Feb 2020].

12 Compton WM, Jones CM, Baldwin GT. Relationship between nonmedical prescription-opioid use and heroin use. N Eng/ J Med Overseas Ed 2016;374:154-63.

13 Brummett CM, Waljee JF, Goesling J, et al. New persistent opioid use after minor and major surgical procedures in US adults. JAMA Surg 2017;152:e170504.

14 Pace M, Gannon K, Friedland M, et al. Influence of inpatient opioid consumption on persistent use following total knee arthroplasty. Reg Anesth Pain Med 2021:46:99-103.

15 Lee JD, Novo P. Comparative effectiveness of extended-release naltrexone versus buprenorphine-naloxone for opioid relapse prevention (X:BOT): a multicentre, openlabel, randomized controlled trial. Lancet 2018

16 Whelan PJ, Remski K. Buprenorphine vs methadone treatment: a review of evidence in both developed and developing worlds. J Neurosci Rural Pract 2012:3:45-50.

17 Mattick RP, Breen C, Kimber J, et al. Buprenorphine maintenance versus placebo or methadone maintenance for opioid dependence. Cochrane Database Syst Rev 2014:CD002207.

18 Hser Y-I, Saxon AJ, Huang D, et al. Treatment retention among patients randomized to buprenorphine/naloxone compared to methadone in a multi-site trial. Addiction 2014;109:79-87.

19 Welsh C, Valadez-Meltzer A. Buprenorphine: a (relatively) new treatment for opioid dependence. Psychiatry 2005;2:29-39.

20 Shulman M, Wai JM, Nunes EV. Buprenorphine treatment for opioid use disorder: an overview. CNS Drugs 2019;33:567-80.

21 Sullivan LE, Fiellin DA. Narrative review: buprenorphine for opioid-dependent patients in office practice. Ann Intern Med 2008;148:662-70

22 DHHS. SAMHSA Medications for Opioid Use Disorder - Full Document. Treatment Improvement Protocol (TIP) Series 63. Publication No. (SMA) PEP20-02-01-006, 2020.

23 Walsh SL, Preston KL, Stitzer ML, et al. Clinical pharmacology of buprenorphine: ceiling effects at high doses. Clin Pharmacol Ther 1994;55:569-80.

24 AHFS $®$ DI Essentials ${ }^{T M}$. American Society of Health-System Pharmacists $®$, 4500 East-West highway, suite 900, Bethesda, MD 20814, 2004

25 U.S. Preventive Services Task Force. Grade definitions. Available: https://www.uspr eventiveservicestaskforce.org/uspstf/about-uspstf/methods-and-processes/gradedefinitions [Accessed 5 Apr 2021].

26 Cohen SP, Bhatia A, Buvanendran A, et al. Consensus guidelines on the use of intravenous ketamine infusions for chronic pain from the American Society of regional anesthesia and pain medicine, the American Academy of pain medicine, and the American Society of Anesthesiologists. Reg Anesth Pain Med 2018;386:1-46.

27 Helm li S, Simopoulos TT, Stojanovic M, et al. Effectiveness of thermal annular procedures in treating discogenic low back pain. Pain Physician 2017;20:447-70.

28 Deer TR, Pope JE, Hayek SM, et al. The Polyanalgesic consensus conference (PacC) recommendations for intrathecal drug delivery: guidance for improving safety and mitigating risks. Neuromodulation 2017:20:155-76.

29 Cohen SP, Wallace MS, Rauck RL. Unique aspects of clinical trials of invasive therapies for chronic pain: an ACTTION evidence-based review. Pain Rep2019;4:e687

30 National Academies of Sciences, Engineering, and Medicine. Medications for opioid use disorder save lives. Washington, DC: The National Academies Press, 2019.

31 Englander $\mathrm{H}$, Weimer $\mathrm{M}$, Solotaroff $\mathrm{R}$, et al. Planning and designing the improving addiction care team (impact) for hospitalized adults with substance use disorder. $J$ Hosp Med 2017;12:339-42.

32 Madras BK, Ahmad NJ, Wen J. The prevention, treatment, and recovery Working group of the action collaborative on countering the U.S. opioid epidemic. NAM perspectives. discussion paper, Washington, DC. Available: https://doi.org/10.31478/ $202004 b$
33 Sharfstein JM, Olsen Y. Making Amends for the opioid epidemic. JAMA 2019:321:1446-7.

34 Yoast RA, Filstead WJ, Wilford BB, et al. Teaching about substance abuse. Virtual Mentor 2008:10:21-9.

35 Mendoza S, Rivera-Cabrero AS, Hansen H. Shifting blame: buprenorphine prescribers, addiction treatment, and prescription monitoring in middle-class America. Transcult Psychiatry 2016;53:465-87.

36 Huskamp HA, Riedel LE, Barry CL, et al. Coverage of medications that treat opioid use disorder and opioids for pain management in marketplace plans, 2017. Med Care 2018:56:505-9.

37 US Department of Health and Human Services. Announcement of practice guidelines for the administration of buprenorphine for treating opioid use disorder. Available: https://www.hhs.gov/sites/default/files/mat-physician-practice-guidelines.pdf [Accessed 8 Feb 2021]

38 Pollini RA, O'Toole TP, Ford D, et al. Does this patient really want treatment? factors associated with baseline and evolving readiness for change among hospitalized substance using adults interested in treatment. Addict Behav 2006:31:1904-18.

39 Coffa D, Harter K, Smith B. Acute pain and perioperative management in opioid use disorder: pain control in patients on buprenorphine, methadone, or naltrexone. SHOUT guidelines. California health care Foundation. Available: https://drive. google.com/drive/folders/1TYvygcY2FcQjvdgEjfvo4JtEvkk0xBe4 [Accessed 20 Sep 2020].

40 Pierce M, Bird SM, Hickman M, et al. Impact of treatment for opioid dependence on fatal drug-related poisoning: a national cohort study in England. Addiction 2016;111:298-308.

41 Birnbaum HG, White AG, Schiller M, et al. Societal costs of prescription opioid abuse, dependence, and misuse in the United States. Pain Med 2011;12:657-67.

42 White AG, Birnbaum HG, Mareva MN, et al. Direct costs of opioid abuse in an insured population in the United States. J Manag Care Pharm 2005;11:469-79.

43 White SR, Bird SM, Merrall ELC, et al. Drugs-related death soon after hospitaldischarge among drug treatment clients in Scotland: record linkage, validation, and investigation of risk-factors. PLoS One 2015;10:e0141073.

44 Larochelle MR, Bernson D, Land T, et al. Medication for opioid use disorder after nonfatal opioid overdose and association with mortality: a cohort study. Ann Intern Med 2018;169:137-45.

45 Poorman E. The Number Needed to Prescribe - What Would It Take to Expand Access to Buprenorphine? N Engl J Med 2021;384:1783-4.

46 Evans E, Li L, Min J, et al. Mortality among individuals accessing pharmacological treatment for opioid dependence in California, 2006-10. Addiction 2015:110:996.

47 Wang S. Historical review: opiate addiction and opioid receptors. Cell Transplant 2019:28:233-8

48 Koehl JL, Zimmerman DE, Bridgeman PJ. Medications for management of opioid use disorder. Am J Health Syst Pharm 2019:76:1097-103.

49 Methadone. DailyMed. US national library of medicine, 2020. Available: https:// dailymed.nlm.nih.gov/dailymed/search.cfm?labeltype=all\&query=methadone [Accessed 8 Feb 2021].

50 Buprenorphine. DailyMed. US national library of medicine, 2020. Available: https:// dailymed.n/m.nih.gov/dailymed/search.cfm?labeltype=all\&query=buprenorphine [Accessed 8 Feb 2021].

51 Naltrexone. DailyMed. US national library of medicine, 2020. Available: https:// dailymed.nlm.nih.gov/dailymed/search.cfm?labeltype=all\&query=naltrexone [Accessed 8 Feb 2021]

52 Ferrari A, Coccia CPR, Bertolini A, et al. Methadone--metabolism, pharmacokinetics and interactions. Pharmacol Res 2004;50:551-9.

53 Gowing L, Ali R, White JM, et al. Buprenorphine for managing opioid withdrawal. Cochrane Database Syst Rev 2017;2:CD002025

54 Mendelson J, Jones RT. Clinical and pharmacological evaluation of buprenorphine and naloxone combinations: why the 4:1 ratio for treatment? Drug Alcohol Depend 2003:70:529-37.

55 Suboxone. Buprenorphine hydrochloride and naloxone hydrochloride dehydrate) sublingual film. prescribing information. Individior, Inc, 2019. Available: https://www. suboxone.com/pdfs/prescribing-information.pdf [Accessed 7 Jul 2021]

56 Virk MS, Arttamangkul S, Birdsong WT, et al. Buprenorphine is a weak partial agonist that inhibits opioid receptor desensitization. J Neurosci 2009:29:7341-8.

57 Campbell ND, Lovell AM. The history of the development of buprenorphine as an addiction therapeutic. Ann N Y Acad Sci 2012;1248:124-39.

58 Raffa RB, Haidery M, Huang H-M, et al. The clinical analgesic efficacy of buprenorphine. J Clin Pharm Ther 2014;39:577-83.

59 Meske DS, Lawal OD, Elder $\mathrm{H}$, et al. Efficacy of opioids versus placebo in chronic pain: a systematic review and meta-analysis of enriched enrollment randomized withdrawal trials. J Pain Res 2018;11:923-34

60 Payandemehr $\mathrm{P}$, Jalili M, Mostafazadeh Davani B, et al. Sublingual buprenorphine for acute renal colic pain management: a double-blind, randomized controlled trial. Int J Emerg Med 2014;7:1.

61 Koppert W, Ihmsen H, Körber N, et al. Different profiles of buprenorphine-induced analgesia and antihyperalgesia in a human pain model. Pain 2005;118:15-22.

62 Coluzzi F, Bifulco F, Cuomo A, et al. The challenge of perioperative pain management in opioid-tolerant patients. Ther Clin Risk Manag 2017;13:1163-73. 
63 Lembke A, Ottestad E, Schmiesing C. Patients maintained on buprenorphine for opioid use disorder should continue buprenorphine through the perioperative period. Pain Med 2019;20:425-8.

64 Matthews S, Dwyer R, Snoek A. Stigma and Self-Stigma in addiction. J Bioeth Ing 2017; 14:275-86.

65 Ward EN, Quaye AN-A, Wilens TE. Opioid use disorders: perioperative management of a special population. Anesth Analg 2018;127:539-47.

66 Committee on Standards and Practice Parameters, Apfelbaum JL, Connis RT, et al. Practice Advisory for preanesthesia evaluation: an updated report by the American Society of Anesthesiologists Task force on Preanesthesia evaluation. Anesthesiology 2012;116:522-38.

67 Smith PC, Schmidt SM, Allensworth-Davies D, et al. A single-question screening test for drug use in primary care. Arch Intern Med 2010;170:1155-60.

68 Skinner HA. The drug abuse screening test. Addict Behav 1982;7:363-71.

69 Yudko E, Lozhkina O, Fouts A. A comprehensive review of the psychometric properties of the drug abuse screening test. I Subst Abuse Treat 2007:32:189-98.

70 Volkow ND, Koob GF, McLellan AT. Neurobiologic advances from the brain disease model of addiction. N Engl J Med 2016:374:363-71.

71 Tsui Jl, Lira MC, Cheng DM, et al. Chronic pain, craving, and illicit opioid use among patients receiving opioid agonist therapy. Drug Alcohol Depend 2016;166:26-31.

72 Larson MJ, Paasche-Orlow M, Cheng DM, et al. Persistent pain is associated with substance use after detoxification: a prospective cohort analysis. Addiction 2007:102:752-60.

73 Anderson TA, Quaye ANA, Ward EN, et al. To stop or not, that is the question: acute pain management for the patient on chronic buprenorphine. Anesthesiology 2017;126:1180-6.

74 Center for Substance Abuse Treatment Clinical Guidelines for the Use of Buprenorphine in the Treatment of Opioid Addiction. Treatment improvement protocol (tip) series 40. DHHS publication No. (SMA) 04-3939. Rockville, MD: Substance Abuse and Mental Health, 2004.

75 Macintyre PE, Russell RA, Usher KAN, et al. Pain relief and opioid requirements in the first 24 hours after surgery in patients taking buprenorphine and methadone opioid substitution therapy. Anaesth Intensive Care 2013:41:222-30.

76 Kornfeld H, Manfredi L. Effectiveness of full agonist opioids in patients stabilized on buprenorphine undergoing major surgery: a case series. Am J Ther 2010;17:523-8.

77 Silva MJ, Rubinstein A. Continuous perioperative sublingual buprenorphine. J Pain Palliat Care Pharmacother 2016;30:289-93.

78 Vilkins AL, Bagley SM, Hahn KA, et al. Comparison of post-cesarean section opioid analgesic requirements in women with opioid use disorder treated with methadone or buprenorphine. J Addict Med 2017;11:397-401.

79 Silverman SM. Opioid induced hyperalgesia: clinical implications for the pain practitioner. Pain Physician 2009;12:679-84.

80 Mercadante S, Villari P, Ferrera P, et al. Safety and effectiveness of intravenous morphine for episodic breakthrough pain in patients receiving transdermal buprenorphine. J Pain Symptom Manage 2006:32:175-9.

81 American Society of Addiction Medicine. National practice guideline for the treatment of opioid use disorder. Available: https://www.asam.org/docs/defaultsource/quality-science/npg-jam-supplement.pdf?sfvrsn=a00a52c2_2 [Accessed 22 Sep 2020].

82 Bentzley BS, Barth KS, Back SE, et al. Discontinuation of buprenorphine maintenance therapy: perspectives and outcomes. J Subst Abuse Treat 2015;52:48-57.

83 Goel A, Azargive S, Lamba W. The perioperative patient on buprenorphine: a systematic review of perioperative management strategies and patient outcomes. Le patient en période périopératoire sous buprénorphine: revue systématique des stratégies de gestion périopératoire et de l'évolution des patients. Can J Anaesth 2019;66:201-17.

84 Warner NS, Warner MA, Cunningham JL, et al. A practical approach for the management of the mixed opioid agonist-antagonist buprenorphine during acute pain and surgery. Mayo Clin Proc 2020;95:1253-67.

85 Greenwald MK, Johanson C-E, Moody DE, et al. Effects of buprenorphine maintenance dose on mu-opioid receptor availability, plasma concentrations, and antagonist blockade in heroin-dependent volunteers. Neuropsychopharmacology 2003:28:2000-9.

86 Greenwald MK, Comer SD, Fiellin DA. Buprenorphine maintenance and mu-opioid receptor availability in the treatment of opioid use disorder: implications for clinical use and policy. Drug Alcohol Depend 2014;144:1-11.

87 Zubieta J, Greenwald MK, Lombardi U, et al. Buprenorphine-induced changes in muopioid receptor availability in male heroin-dependent volunteers: a preliminary study. Neuropsychopharmacology 2000;23:326-34

88 Quaye AN-A, Zhang Y. Perioperative management of buprenorphine: solving the conundrum. Pain Med 2019;20:1395-408

89 Comer SD, Walker EA, Collins ED. Buprenorphine/naloxone reduces the reinforcing and subjective effects of heroin in heroin-dependent volunteers. Psychopharmacology 2005;181:664-75.

90 Greenwald MK, Schuh KJ, Hopper JA, et al. Effects of buprenorphine sublingual tablet maintenance on opioid drug-seeking behavior by humans. Psychopharmacology 2002;160:344-52.
91 Goel A, Azargive S, Weissman JS, et al. Perioperative pain and addiction interdisciplinary network (pain) clinical practice Advisory for perioperative management of buprenorphine: results of a modified Delphi process. $\mathrm{Br} J$ Anaesth 2019:123:e333-42.

92 Book SW, Myrick H, Malcolm R, et al. Buprenorphine for postoperative pain following general surgery in a buprenorphine-maintained patient. Am J Psychiatry 2007:164:979.

93 Chern S-YS, Isserman R, Chen L, et al. Perioperative pain management for patients on chronic buprenorphine: a case report. J Anesth Clin Res 2013;3:1000250.

94 Huang A, Katznelson R, de Perrot $M$, et al. Perioperative management of a patient undergoing Clagett window closure stabilized on Suboxone ${ }^{\circledR}$ for chronic pain: a case report. Can J Anaesth 2014;61:826-31.

95 Stromer W, Michaeli K, Sandner-Kiesling A. Perioperative pain therapy in opioid abuse. Eur J Anaesthesiol 2013;30:55-64.

96 Joo SS, Hunter 00, Tamboli M, et al. Implementation of a patient-specific tapering protocol at discharge decreases total opioid dose prescribed for 6 weeks after elective primary spine surgery. Reg Anesth Pain Med 2020:45:474-8.

97 Tamboli M, Mariano ER, Gustafson KE, et al. A multidisciplinary patient-specific opioid prescribing and tapering protocol is associated with a decrease in total opioid dose prescribed for six weeks after total hip arthroplasty. Pain Med 2020:21:1474-81

98 Katz A, Goldberg D, Smith J. Tobacco, alcohol, and drug use and willingness to change. J Hosp Med 2008;3:369-75

992008 PHS Guideline Update Panel, Liaisons, and Staff. Treating tobacco use and dependence: 2008 update U.S. public health service clinical practice guideline executive summary. Respir Care 2008;53:1217-22.

100 Jonas DE, Garbutt JC, Amick HR, et al. Behavioral counseling after screening for alcohol misuse in primary care: a systematic review and meta-analysis for the U.S. preventive services Task force. Ann Intern Med 2012;157:645-54.

101 D'Onofrio G, O'Connor PG, Pantalon MV, et al. Emergency department-initiated buprenorphine/naloxone treatment for opioid dependence: a randomized clinical trial. JAMA 2015:313:1636-44.

102 Liebschutz JM, Crooks D, Herman D, et al. Buprenorphine treatment for hospitalized, opioid-dependent patients: a randomized clinical trial. JAMA Intern Med 2014:174:1369-76.

103 Hansen LE, Stone GL, Matson CA, et al. Total joint arthroplasty in patients taking methadone or buprenorphine/naloxone preoperatively for prior heroin addiction: a prospective matched cohort study. J Arthroplasty 2016;31:1698-701.

104 Wei J, Defries T, Lozada M, et al. An inpatient treatment and discharge planning protocol for alcohol dependence: efficacy in reducing 30-day readmissions and emergency department visits. J Gen Intern Med 2015:30:365-70.

105 Substance Abuse and Mental Health Services Administration (SAMHSA). Drug abuse warning network, 2011: national estimates of drug-related emergency department visits. Available: http://www.samhsa.gov/data/2k13/DAWN2k11ED/DAWN2k11ED. htm\#5.1 [Accessed 21 May 2020].

106 Patel N, Schwenk ES, Ferd P, et al. An anesthesiologist-led inpatient buprenorphine initiative. Pain Pract 2021;21:692-7.

107 Umbricht A, Hoover DR, Tucker MJ, et al. Opioid detoxification with buprenorphine, clonidine, or methadone in hospitalized heroin-dependent patients with HIV infection. Drug A/cohol Depend 2003:69:263-72.

108 Ravndal E, Amundsen EJ. Mortality among drug users after discharge from inpatient treatment: an 8-year prospective study. Drug Alcohol Depend 2010;108:65-9.

109 Weiner SG, Baker O, Bernson D, MPH Baker MD MD, PhD BD, et al. One year mortality of patients treated with naloxone for opioid overdose by emergency medical services. Subst Abus 2020:1-5

110 Doran KM, Rahai N, McCormack RP, et al. Substance use and homelessness among emergency department patients. Drug Alcohol Depend 2018;188:328-33.

111 Substance Abuse and Mental Health Services Administration (SAMHSA). Specia circumstances for providing buprenorphine, 2016. Available: https://www.samhsa. gov/medication-assisted-treatment/legislation-regulations-guidelines/specialcircumstances-providing-buprenorphine [Accessed 21 May 2020].

112 Cornish R, Macleod J, Strang J, et al. Risk of death during and after opiate substitution treatment in primary care: prospective observational study in UK general practice research database. BMJ 2010;341:c5475.

113 Houry DE, Haegerich TM, Vivolo-Kantor A. Opportunities for prevention and intervention of opioid overdose in the emergency department. Ann Emerg Med 2018:71:688-90.

114 American College of Emergency Physicians. State legislation requiring emergency medication assisted therapy for opioid use disorder. Available: https://www.acep.org/ how-we-serve/sections/toxicology/news/september-2018/state-legislation-requiringemergency-medication-assisted-therapy-for-opioid-use-disorder/ [Accessed $5 \mathrm{Apr}$ 2021]

115 Kaucher KA, Caruso EH, Sungar G, et al. Evaluation of an emergency department buprenorphine induction and medication-assisted treatment referral program. Am J Emerg Med 2020;38:300-4.

116 Vestal C. Few doctors are willing, able to prescribe powerful anti-addiction drugs, 2016. Available: http://www.pewtrusts.org/en/research-and-analysis/blogs/stateline/ 
2016/01/15/few-doctors-are-willing-able-to-prescribe-powerful-anti-addiction-drugs [Accessed 5 Apr 2021].

117 Randhawa PA, Brar R, Nolan S. Buprenorphine-naloxone "microdosing": an alternative induction approach for the treatment of opioid use disorder in the wake of North America's increasingly potent illicit drug market. Can Med Assoc J 2020;192:E73.

118 Hämmig R. Einleitung einer Substitutionsbehandlung mit Buprenorphin unter vorübergehender Überlappung mit Heroinkonsum: ein neuer Ansatz („Berner Methode"). Suchttherapie2010;11:129-32.

119 Hämmig R, Kemter A, Strasser J, et al. Use of microdoses for induction of buprenorphine treatment with overlapping full opioid agonist use: the Bernese method. Subst Abuse Rehabil 2016;7:99-105.

120 Klaire S, Zivanovic R, Barbic SP, et al. Rapid micro-induction of buprenorphine/ naloxone for opioid use disorder in an inpatient setting: a case series. Am J Addict 2019;28:262-5.

121 Moe J, Badke K, Pratt M. Microdosing and standard-dosing take-home buprenorphine from the emergency department: a feasibility study. J Am Coll Emerg Physicians Open 2020

122 Herring AA, Perrone J, Nelson LS. Managing opioid withdrawal in the emergency department with buprenorphine. Ann Emerg Med 2019;73:481-7.

123 ToolkitsE-QO. Available: https://www.acep.org/administration/quality/equal/ emergency-quality-network-e-qual/e-qual-opioid-initiative/e-qual-opioid-toolkit/ [Accessed 13 Mar 2021].

124 National Institute of Drug Abuse. Initiating buprenorphine treatment in the emergency department. Available: https://www.drugabuse.gov/nidamed-medicalhealth-professionals/discipline-specific-resources/emergency-physicians-firstresponders/initiating-buprenorphine-treatment-in-emergency-department [Accessed 5 Apr 2021].

125 Jaeger S, Fuehrlein B. Buprenorphine initiation to treat opioid use disorder in emergency rooms. J Neurol Sci 2020;411:116716.

126 CA bridge. Available: https://cabridge.org/resource/buprenorphine-bup-hospitalquick-start/ Buprenorphine Hospital Quick Start [Accessed 5 Apr 2021].

127 Wolff RF, Aune D, Truyers C, et al. Systematic review of efficacy and safety of buprenorphine versus fentanyl or morphine in patients with chronic moderate to severe pain. Curr Med Res Opin 2012;28:833-45.

128 American College of Emergency Physicians Clinical Policies Subcommittee (Writing Committee) on Opioids, Hatten BW, Cantrill SV, et al. Clinical policy: critical issues related to opioids in adult patients presenting to the emergency department. Ann Emerg Med 2020;76:e13-39.

129 Kampman K, Jarvis M. American Society of addiction medicine (ASAM) national practice guideline for the use of medications in the treatment of addiction involving opioid use. J Addict Med 2015;9:358-67.

130 Kinnunen $\mathrm{M}$, Piirainen $\mathrm{P}$, Kokki $\mathrm{H}$, et al. Updated clinical pharmacokinetics and pharmacodynamics of oxycodone. Clin Pharmacokinet 2019;58:705-25.

131 Hill HF, Coda BA, Tanaka A, et al. Multiple-dose evaluation of intravenous hydromorphone pharmacokinetics in normal human subjects. Anesth Analg 1991;72:330???336-6.

132 Cushman PA, Liebschutz JM, Anderson BJ, et al. Buprenorphine initiation and linkage to outpatient buprenorphine do not reduce frequency of injection opiate use following hospitalization. J Subst Abuse Treat 2016;68:68-73.

133 Suzuki J, DeVido J, Kalra I, et al. Initiating buprenorphine treatment for hospitalized patients with opioid dependence: a case series. Am J Addict 2015;24:10-14.

134 Duber HC, Barata IA, Cioè-Peña E, et al. Identification, management, and transition of care for patients with opioid use disorder in the emergency department. Ann Emerg Med 2018:72:420-31.

135 Substance abuse and mental health services administration (SAMHSA) buprenorphine practitioner locator. Available: https://www.samhsa.gov/medicationassisted-treatment/practitioner-program-data/treatment-practitioner-locator [Accessed 6 Apr 2021].

136 Ahmed OM, Mao JA, Holt SR, et al. A scalable, automated warm handoff from the emergency department to community sites offering continued medication for opioid use disorder: lessons learned from the embed trial stakeholders. J Subst Abuse Treat 2019:102:47-52

137 National alliance of Advocates for buprenorphine treatment treatment match. Available: https://www.treatmentmatch.org/ [Accessed 5 Apr 2021].

138 Kelly T, Hoppe JA, Zuckerman M, et al. A novel social work approach to emergency department buprenorphine induction and warm hand-off to community providers. Am J Emerg Med 2020;38:1286-90.

139 Martin A, Butler K, Chavez T, et al. Beyond buprenorphine: models of follow-up care for opioid use disorder in the Emergeny department. West J Emerg Med 2020;21:257-63.
140 Ling W, Amass L, Shoptaw S, et al. A multi-center randomized trial of buprenorphinenaloxone versus clonidine for opioid detoxification: findings from the National Institute on drug abuse clinical trials network. Addiction 2005;100:1090-100.

141 Title 21 code of federal regulations. \$1306.07. administering or dispensing of narcotic drugs, 2020. Available: https://www.govinfo.gov/app/details/CFR-2020title21-vol9/CFR-2020-title21-vol9-sec1306-07

142 Cabridge.org. Best practices: inpatient and order set guideline. Available: https:// static1.squarespace.com/static/5c412ab755b02cec3b4ed998/t/5dc2504c449ab611 55c0b1d1/1573015629142/CA+Bridge+-+Best+Practices+-+Inpatient+and+ order+sets+-+NOV+2019.pdf [Accessed 3 Jun 2021]

143 Federal Registrar. Federal guidelines on the for the administration of buprenorphine for treating OUD. Available: https://www.federalregister.gov/documents/2021/04/ 28/2021-08961/practice-guidelines-for-the-administration-of-buprenorphine-fortreating-opioid-use-disorder [Accessed 28 Apr 2021]

144 Centers for Disease Control and Prevention. Emergency preparedness and response. Available: https://emergency.cdc.gov/han/2020/han00438.asp [Accessed 28 Apr 2021].

145 Substance Abuse and Mental Health Services Administration (SAMHSA). Medication assisted treatment. buprenorphine, 2016. Available: https://www.samhsa.gov/ medication-assisted-treatment/medications-counseling-related-conditions/ buprenorphine [Accessed 5 Apr 2021].

146 Jasinski DR, Pevnick JS, Griffith JD. Human pharmacology and abuse potential of the analgesic buprenorphine: a potential agent for treating narcotic addiction. Arch Gen Psychiatry 1978:35:501-16.

147 Wightman RS, Perrone J, Scagos R, et al. Opioid overdose deaths with buprenorphine detected in postmortem toxicology: a retrospective analysis. J Med Toxicol 2021;17:10-15

148 Marcucci C, Fudin J, Thomas P, et al. A new pattern of buprenorphine misuse may complicate perioperative pain control. Anesth Analg 2009;108:1996-7.

149 Brummett CM, Trivedi KA, Dubovoy AV, et al. Dexmedetomidine as a novel therapeutic for postoperative pain in a patient treated with buprenorphine. J Opioid Manag 2009:5:175-9.

150 Rodgman C, Pletsch G. Double successful buprenorphine/naloxone induction to facilitate cardiac transplantation in an iatrogenically opiate-dependent patient. $J$ Addict Med 2012:6:177-8.

151 McCormick Z, Chu SK, Chang-Chien GC, et al. Acute pain control challenges with buprenorphine/naloxone therapy in a patient with compartment syndrome secondary to McArdle's disease: a case report and review. Pain Med 2013:14:1187-91.

152 Israel JS, Poore SO. The clinical conundrum of perioperative pain management in patients with opioid dependence: lessons from two cases. Plast Reconstr Surg 2013:131:657e-8.

153 Khelemsky Y, Schauer J, Loo N. Effect of buprenorphine on total intravenous anesthetic requirements during spine surgery. Pain Physician 2015;18:E261-4.

154 Jones HE, Johnson RE, Milio L. Post-cesarean pain management of patients maintained on methadone or buprenorphine. Am J Addict 2006;15:258-9.

155 Höflich AS, Langer $M$, Jagsch $R$, et al. Peripartum pain management in opioid dependent women. Eur J Pain 2012;16:574-84.

156 Mehta D, Thomas V, Johnson J, et al. Continuation of buprenorphine to facilitate postoperative pain management for patients on buprenorphine opioid agonist therapy. Pain Physician 2020;23:E163-4.

157 Alford DP, Compton P, Samet JH. Acute pain management for patients receiving maintenance methadone or buprenorphine therapy. Ann Intern Med 2006;144:127-34

158 Childers JW, Arnold RM. Treatment of pain in patients taking buprenorphine for opioid addiction \#221. J Palliat Med 2012;15:613-4.

159 Bryson EO. The perioperative management of patients maintained on medications used to manage opioid addiction. Curr Opin Anaesthesiol 2014;27:359-64.

160 Sen S, Arulkumar S, Cornett EM, et al. New pain management options for the surgical patient on methadone and buprenorphine. Curr Pain Headache Rep 2016;20:16.

161 Jonan AB, Kaye AD, Urman RD. Buprenorphine formulations: clinical best practice strategies recommendations for perioperative management of patients undergoing surgical or interventional pain procedures. Pain Physician 2018;21:E1-12.

162 Harrison TK, Kornfeld H, Aggarwal AK, et al. Perioperative considerations for the patient with opioid use disorder on buprenorphine, methadone, or naltrexone maintenance therapy. Anesthesiol Clin 2018;36:345-59.

163 Berry P. Besio S, Brooklyn J. Vermont practice guidelines 2015; Vermont department of health; division of alcohol and drug abuse programs. Available: http:// contentmanager.med.uvm.edu/docs/default-source/vchip-documents/vchip 2buprenorphine_guidelines.pdf?sfvrsn $=2$ [Accessed 14 Mar 2021]. 